\title{
Constructing QFT's wherein Lorentz Invariance is broken by dissipative effects in the UV
}

\section{R. Parentani $* \dagger$}

Laboratoire de Physique Théorique, UMR 8627,

Université Paris-Sud 11, Bât. 210,

91405 Orsay Cedex, France

E-mail: parentadth.u-psud.fr

\begin{abstract}
There has been a recent interest in considering Quantum Field Theories in which Lorentz Invariance is broken in the UV sector. However attention has been mostly limited to dispersive theories. In this work we provide the generalized settings for studying dissipation. Unitarity is preserved by coupling the original fields to additional (heavy) fields which induce the dissipation. Starting with Lagrangians breaking LI in the UV, we learn that dissipative effects unavoidably develop in the effective theory. We then covariantize these Lagrangians in order to address the trans-Planckian question of inflation and black hole physics. The peculiar properties of the additional fields inducing dissipation is revealed by the covariantization. The links with the phenomenological approach to Quantum Gravity and with some Brane World scenarios are also discussed.
\end{abstract}

From Quantum to Emergent Gravity: Theory and Phenomenology

June 11-15 2007

Trieste, Italy

\footnotetext{
* Speaker.

$\dagger$ This work contains large extracts of the manuscript entitled: Confronting the trans-Planckian question of inflationary cosmology with dissipative effects.
} 


\section{Introduction}

Even though relativistic QFT provides an excellent description of particle physics, being noncompact, Lorentz symmetry has been tested only up to a certain energy scale[1]. Thus one cannot exclude the possibility that some high energy processes break the invariance under boosts, thereby introducing a threshold energy $\Lambda_{L V}$, and a preferred frame. It is therefore of interest to understand what would be the signatures of QFTs wherein this possibility is realized.

Unlike dispersive effects which have been studied in details [2], dissipative effects have so far received much less attention. In this work we provide the grounds for this extension. To this end, we shall first construct QFT displaying dissipative effects in the UV. Indeed, to handle dissipation requires settings which are wider than those of relativistic and dispersive QFTs because, if one introduces dissipation from the outset in the usual settings, one looses both unitarity and predictability. To preserve them, we shall therefore work with Hamiltonian theories in which dissipative effects are caused by interactions with additional degrees of freedom. Doing so, we shall discover that dissipative effects are generic. That is, when starting with a bare Lagrangian in which $\mathrm{LI}$ is broken by some kinetical or interaction term in the UV, the effective theory (the generating functional) unavoidably develops dissipation above a certain energy scale, simply because nothing can prevent this. (With relativistic QFT instead, LI did prevent it). We thus learn that it is illegitimate to deal with purely dispersive QFT, since these do not satisfy Kramer's relations.

Being engaged in a procedure of generalization, we should have a clear idea of our motivations, aims, and requirements. We first present these aspects.

\subsection{Two requirements}

\section{$1^{\text {st }}$. Unitarity}

As already mentioned, we require that our QFT evolve unitarily. This requirement implies that the dissipative effects be produced by the Hamiltonian dynamics of the entire system. In other words, we shall introduce additional degrees of freedom called $\Psi$, which play the role of an environment, and couple them to the original field $\phi$ in such a way that the latter develops dissipative effects. This means that the (dressed) two-point function of $\phi$ will be given by the usual QM trace

$$
G_{W}(x, y)=\operatorname{Tr}\left[\hat{\rho}_{T} \hat{\phi}(x) \hat{\phi}(y)\right]
$$

where $\hat{\rho}_{T}$ is the initial matrix density of the entire system $\Psi+\phi$, where $\hat{\phi}(x)$ is the Heisenberg field operator evolved with the time ordered exponential of the total Hamiltonian, and where the trace is taken over both $\Psi$ and $\phi$.

\section{$2^{d}$. Stationarity and homogeneity}

Our second requirement concerns the properties of dissipative effects. When considering the theory in vacuum and in Minkowski space-time, we impose that the dissipative effects become significant only above a critical energy $\Lambda_{L V}$, and that they preserve the stationary, the homogeneity, and isotropy of flat space-time. In this case, dissipative effects define a preferred frame which is inertial and globally defined. 
Then, irrespectively of the properties of the additional degrees of freedom $\Psi$ and their interactions with $\phi$, the Fourier transform of the retarded Green function $G_{R}(x, y)$, which is given by $G_{R}(x, y)=\theta\left(t_{x}-t_{y}\right) 2 \operatorname{Im} G_{W}(x, y)$, where $G_{W}$ is the Wightman function of eq. (1.1), is of the form

$$
G_{R}(\omega, \vec{p})=\frac{-i}{\left(-\omega^{2}+p^{2}+\Sigma_{R}(\omega, p)\right)} .
$$

In the true vacuum, at the level of the $2 \mathrm{pt}$ functions, the dissipative (dispersive) effects are indeed completely characterized by the imaginary and odd (real and even) part in $\omega$ of the (retarded) self-energy $\Sigma_{R}(\omega, p)$.

In these expressions, the energy $\omega$ and the spatial momentum square $p^{2}$ have been defined in the preferred frame. To prepare the covariantization of our theory, and therefore its extension to curved space-time, it is usefull to characterize the preferred frame in a coordinate invariant way by a unit time-like vector field, here after called $l^{\mu}$. Then $\omega$ and $p^{2}$ are given by

$$
\omega \equiv l^{\mu} p_{\mu}, \quad p^{2} \equiv \perp^{\mu v} p_{\mu} p_{v}
$$

where $\perp^{\mu v} \equiv \eta^{\mu v}+l^{\mu} l^{v}$ is the (positive definite) metric in the spatial sections orthogonal to $l^{\mu}$.

The novelty is that $\Sigma_{R}$ is a function of $\omega$ and $p$ separately, and not only of the relativistic invariant $\omega^{2}-p^{2}$ as it is the case in relativistic QFT. When $\Sigma$ depends on both $\omega$ and $p$, dissipation can become significant above a critical energy on the mass shell, i.e. along the minima of the denominator of eq. (1.2); a possibility forbidden in LI theories.

With the observation of the simplicity of eq. (1.2), we understand that most of the properties of $\Psi$ will be irrelevant when restricting attention to observables built with only $\phi$. In other words, the effective action of $\phi$ only retains little information about $\Psi$ in its moments, the second of which being the self-energy $\Sigma$. This is very important, because we shall exploit this arbitrariness by choosing the simplest models of $\Psi$ which deliver the required properties of $\Sigma$.

\subsection{Aims and Motivations}

\subsubsection{The phenomenology and the links with Quantum Gravity}

We first aim to describe the phenomenology of dissipative effects in Minkowski space-time and in vacuo. This is rather easy. From eq. (1.2) we understand that the phenomenology of dissipative effects respecting our (minimal) restrictions will be governed by a limited set of functions. In fact, at the level of 2-pt functions, the imaginary part of $\Sigma$ is the only relevant quantity. One easily verifies that the following self-energies induce significant dissipation only above $\Lambda_{L V}$

$$
\operatorname{Im} \Sigma_{R}^{(n)}(\omega, p)=-\frac{\omega}{\Lambda_{L V}} p^{2}\left(\frac{p^{2}}{\Lambda_{L V}^{2}}\right)^{n}
$$

Later in the text, we shall provide Lagrangians of $\Psi$ and $\phi$ which produce this class of imaginary self-energies. We can already relate this class to the set of non-linear dispersion relations which have been used in phenomenological studies:

$$
\omega^{2}=F_{n}^{2}\left(p^{2}\right)=p^{2} \pm p^{2}\left(\frac{p^{2}}{\Lambda_{L V}^{2}}\right)^{n}+O\left(\left(p^{2}\right)^{n+2}\right)
$$


The $+(-)$ sign gives superluminous (subluminous) propagation. It is also worth noticing that a phenomenological study of dispersive and dissipative effects taken toghether can be done with eq. (1.2) by considering both $\operatorname{Re} \Sigma_{R}$ and $\operatorname{Im} \Sigma_{R}$ non zero.

Even though Quantum Gravity is not part of our main concerns, let us say a few words about the relations with our work. Quantum Gravity, whatever version is adopted, implies that the smooth manifold of GR should be replaced by a new structure when reaching a UV threshold energy, call it $\Lambda_{L V}$. When adopting a phenomenological point of view, there is a shift in the interest. The question is no longer: What is this new structure ? but rather: How would it manifest itself in observables ? That is to say: What are the new expressions of the $n$-point correlation functions ? Remember that the predictions of a QFT are all based on its correlation functions.

The simplest of these are the 2-point functions. In whatever replaces the Minkowski manifold, spatial homogeneity and stationarity will be preserved in the mean, (otherwise that version of QG does not describe our world). This implies that the Fourier transform of the "true" Feynman Green function ${ }^{1}$ will belong to the class of functions given in eq. $(1.2){ }^{2}$ (Of course LI might still be respected in which case the self-energy induced by QG will only be a function of $\omega^{2}-p^{2}$.)

The link between our approach and the phenomenological approach to QG which consists in parametrizing its effects (rather than computing them from first principles) is clear: Since we provide the general expression of the 2pt function compatible with QM, in the sense that the Equal Time Commutations relations be still satisfied, our expressions can also be used in a phenomenological QG perspective.

\subsubsection{Mode creation in expanding universes}

When assuming that LI is broken in the UV in Minkowski space, one can then also assume that the density of degrees of freedom, or modes, is finite and of the order of $\Lambda_{L V}^{3}$. (This density should

\footnotetext{
${ }^{1}$ Whatever QG may be, this function will be given by an expression similar to eq. (1.1) with the trace taken over the "true" degrees of freedom. For the benefit of the reader, I wish to add here a remark made by a referee concerning this sentence and my reply to it: "While of course one might claim this is a plausible scenario, one might as well conjecture that in no way transplanckian physics can be described by a quantum field theory of any sort (e.g. QM could be emergent as well)." My answer is twofold. First, I wrote "similar" precisely to leave open the possibility that the trace be not "the usual QM" one. Second, even if QM is only emergent, when considering the two-point function of accessible (effective) degrees of freedom, as $\phi$ in the present case, (like the two-point functions of the phonon field in a BEC), the true expression will, at least, contain a trace (to average out the true configurations so that the correlation function only depends on the arguments of the two operators), a state function (to weigh these configurations), and two operators (which might be complicate composite objects), because, in the IR, the two-point function must behave according to $Q M$. The beauty of two-points correlation functions is that they are c-number functions depending only $\omega$ and $\mathbf{p}$, both in the effective description and in the true description. Therefore a comparison of two versions directly delivers the relevant modifications, see [B] for a comparison of 2pt functions obtained using a QFT in a fixed background and the corresponding ones obtained by studying the solutions of the Wheeler-DeWitt equation.

${ }^{2}$ It has been claimed that QG might turn out to be a purely topological theory because reparametrization invariance would restrict the true observables to be topological charges. If this could be correct in pure $2+1 \mathrm{QG}$ [4], it is harder to conceive the meaning of this statement when applied to $4 \mathrm{D}$, both from a phenomenological and a theoretical point of view. If it were true, it would imply that all quantum transitions, scattering events, ..., we observe are in fact topological invariants (or some part thereof) when properly dressed by QG. This is at best irrelevant. At a theoretical level, the quantum transitions of (heavy) atoms exchanging photons are governed by the Green functions of the radiation field evaluated where the atoms sit, given their wave function. This should remain true when including QG effects, see [ [ ] for a description of atomic transitions based on solutions of the WDW equation wherein topology plays no role.
} 
not be confused with the density of quanta which is always finite in decent quantum states, the "Hadamard" states [6, 7].) This option was not available as long as LI prevailed, and was in fact the source of the UV divergences. If the cutoff $\Lambda_{L V}$ is much higher than the typical frequencies involved in the observables we have access to, it is rather easy to show that the expectation value of these observables will not be significantly affected by $\Lambda_{L V}$, a simple example which can be worked out explicitely is furnished by the Casimir effect. Therefore, in Minkowski space, one cannot expect any significant deviation induced by having cutoff the mode density.

On the contrary, it is more challenging to consider how would such a theory behave in an expanding universe. Indeed one faces an alternative. Either the total number of degrees of freedom in a given comoving volume would stay fixed (or nearly fixed), and therefore the density would decrease like the inverse of the proper volume, or it is the density that would stay (nearly) fixed, and in this case, the number of degrees of freedom would grow linearly with the proper volume, thereby implying mode creation.

The first alternative seems already excluded because the volume of our visible universe increased at least by a factor of $e^{360}$ since the onset of inflation (360 $=60$ e-foldings during inflation $\times$ two for the radiation era $\times$ three because of 3D). Indeed either the density was absurdly high at the onset of inflation, or if the intial density was decent, we should today be lacking degrees of freedom. Moreover if it were true, one could measure the growth of the scale factor by probing locally the vacuum, thereby violating the Equivalence Principle. So, once having assumed a finite density in flat space, we are left with the conclusion that mode creation is unavoidable in expanding universes, and therefore in any curved background geometry.

When adopting this second alternative, two questions should be confronted:

How to describe mode creation in QFT ?

What fixes the state of the newly born modes?

Our motivation is to confront these two questions in the presence of dissipative effects. To this end we first need to extend our QFTs to curved space times.

The main principle we adopt to covariantize our Lagrangians is the Equivalence Principle (or more precisely its extension in the presence of the unit vector field $\left.l^{\mu}\right){ }^{3}$ That is, the Lagrangians will be a sum of scalar functions of the four local fields $\phi, \Psi, g_{\mu v}$ and $l^{v}$ which reduce locally to their Minkowski value in the zero curvature limit. This principle fixes the action density (up to the possibility of some non-minimal coupling) and determines the tensorial nature of the $\Psi$ fields. As explained in the text, they contain a dense set of local degrees of freedom at rest with the vector field $l^{\mu}$.

When covariantization is done, without fine tuning nor additional hypothesis, we shall see that our QFTs are such that, as the universe expands, underdamped modes emerge from overdamped

\footnotetext{
${ }^{3}$ To obtain dissipative QFTs, we could have searched for inspiration in condensed matter models wherein $L I$ is broken in the UV. We have chosen not to pursue this approach for several reasons. First it distances us form our aims, as it requires first understanding the physics at play in the condensed matter analog model, and then explaining its relevance to the problems which interest us. Second this approach also lacks generality and thus hides the steps necessary to construct dissipative models. Third, the deepest reason comes from the Equivalence Principle considered in expanding universes. Indeed any QFT emerging from a discrete structure, e.g. a set of atoms, is bound to fail to preserve the Equivalence Principle after a large number of e-foldings. We see no way to espace this conclusion, besides either modifying QM, or assuming that the expansion of the universe does not affect the "atoms", two options so radical that it seems hopeless to get an acceptable phenomenolgy when adopting them.
} 
modes -thereby effectively describing the creation of propagating degrees of freedom- in such a way that their (proper) density stays constant. Moreover, unlike in former attempts to describe "mode creation" [8, 9], there is no need to supply an extra condition to fix the state of the newly born modes. Indeed, since our models are Hamiltonian, all the information is contained in the initial density matrix $\hat{\rho}_{T}$.

\subsubsection{The trans-Planckian question}

In inflationary cosmology, the primordial density fluctuations arise from the amplification of vacuum fluctuations which had very short wave lengths (very large proper frequencies) at the onset of inflation[10]. Similarly, Hawking radiation emitted by a black hole emerges from configurations which had extremely high initial frequencies[11]. In both cases, the unbounded frequency growth questions the validity of the predictions because these have been obtained using the standard treatment, namely some quantum field propagating in a curved space time. However there is no reason to believe that these settings still provide a reliable approximation at frequencies way above or even near the Planck scale.

Following original work of Unruh and Jacobson [12, 13, the robustness of the standard predictions against modifying the theory in the UV have been tested by introducing dispersion relations which become non linear above a certain UV scale $\Lambda_{L V}$, see eq. (1.5). Even though the propagation of the configurations is severely modified when this scale is reached, it was shown that the properties are essentially unmodified when the two relevant scales are well separated, i.e. in BH physics[14] when $\kappa / \Lambda_{L V} \ll 1$ where $\kappa$ is the surface gravity, and in inflation[15, 16] when $H / \Lambda_{L V} \ll 1$ where $H$ is the Hubble parameter. What guarantees the robustness is that the vacuum state evolves adiabatically.

Our aim is to generalize these works by providing dissipative models in which the power spectrum (and Hawking radiation) can be computed. These models are the same as those of the former subsection. The fact that the Equivalence Principle is preserved will guarantee, as we shall see, the adiabaticity of the evolution of the true vacuum as long as the gradients of the metric are much smaller than the UV scale $\Lambda_{L V}$.

To conclude, we would like bring to the reader's attention to the following remarks. First, to compute the power spectrum in inflationary models, and the asymptotic properties of Hawking radiation, it is sufficient to know the $2 \mathrm{pt}$ function of eq. (1.1). Indeed, one always has

$$
\begin{aligned}
P_{k}(t) & \equiv \int d^{3} x e^{-i \mathbf{k x}} G_{a}(t, \mathbf{x} ; t, \mathbf{0}), \\
n_{\omega, l, m}+\frac{1}{2} & \equiv \int d t e^{i \omega t} G_{a}(t, r ; 0, r, l, m), \quad r \gg r_{h} .
\end{aligned}
$$

In inflation, the first equality follows from the definition of the power spectrum $P_{k}$ which is given by the spatial Fourier transform of the anti-commutator $G_{a}$ evaluated, at equal time after horizon exit, in the Bunch-Davies vacuum. As of Hawking radiation, $n(\omega, l, m)$, the asymptotic distribution at fixed angular momentum $l, m$, is given by Fourier transform with respect to the asymptotic time of the in-vacuum anti-commutator far away from the hole.

Second, in the presence of dissipation, as in all interacting models, the knowledge of the anticommutator is also necessary. In these cases indeed, the only way to extract the relevant predictions is through the above expressions. 
We have organized the paper as follows. We first construct dissipative theories in Minkowski space-time. In this case, because of stationarity the analysis is simple and instructive. We then covariantize these models and briefly comment on the trans-Planckian question in inflation and in black hole physics. In long Appendices we provide self-contained presentations of the properties of Green functions in the presence of dissipation, and how to use them to cover the phenomenology.

\section{Dissipation in Minkowski space from $L V$ effects}

In this section, we provide a class of models defined in Minkowski space time which exhibit dissipative effects above a certain energy scale $\Lambda_{L V}$. Stationarity, homogeneity and isotropy will be exactly preserved. Therefore, the only invariance of relativistc QFT which is broken is that under boosts. These theories define a preferred rest frame which is globally defined, as it is the case of FLRW space-times. Even though a covariant description exists, for simplicity of the presentation, we first work in that frame. At the end of this Section we shall covariantize to prepare the extension to curved space-times.

\subsection{Free field settings}

We start with a brief presentation of the free field description to introduce notations and to point out what are the properties which are lost in the presence of dissipation.

The action of our free massless field $\phi$ is the usual one:

$$
S_{\phi}=\frac{1}{2} \int d t d^{3} x\left(\partial_{t} \phi^{2}-\partial_{\mathbf{x}} \phi \cdot \partial_{\mathbf{x}} \phi\right),
$$

where $t$ and $\mathbf{x}$ are Cartesian coordinates. Due to the homogeneity of space, the equation of motion can be analyzed mode by mode:

$$
\phi(t, \mathbf{x})=\int \frac{d^{3} p}{(2 \pi)^{3 / 2}} e^{i \mathbf{p} \cdot \mathbf{x}} \phi_{\mathbf{p}}(t)
$$

The mode operator $\phi_{\mathbf{p}}(t)$ obeys

$$
\left(\partial_{t}^{2}+\omega_{p}^{2}\right) \phi_{\mathbf{p}}=0
$$

where $\omega_{p}^{2}=p^{2}=\mathbf{p} \cdot \mathbf{p}$ is the standard relativistic dispersion relation. Notice that eq. (2.3) is second order, homogeneous (no source term), and time reversible (no odd power of $\partial_{t}$ ), three properties we shall loose when introducing interactions breaking $L I$.

In homogeneous space-times, the canonical Equal Time Commutator of the field and its momentum implies that $\phi_{\mathbf{p}}(t)$ the Fourier transform of the field operator obeys

$$
\left[\phi_{\mathbf{p}}(t), \partial_{t} \phi_{\mathbf{p}^{\prime}}^{\dagger}(t)\right]=i \delta^{3}\left(\mathbf{p}-\mathbf{p}^{\prime}\right)
$$

When decomposing the mode operator as

$$
\phi_{\mathbf{p}}(t)=a_{\mathbf{p}} \phi_{p}(t)+a_{-\mathbf{p}}^{\dagger} \phi_{p}^{*}(t),
$$

where the destruction and creation operators satisfy the usual commutators

$$
\left[a_{\mathbf{p}}, a_{\mathbf{p}^{\prime}}^{\dagger}\right]=\delta^{3}\left(\mathbf{p}-\mathbf{p}^{\prime}\right), \quad\left[a_{\mathbf{p}}, a_{\mathbf{p}^{\prime}}\right]=0,
$$


eq. (2.4) is verified at all times because the Wronskian of the positive frequency (c-number) mode

$$
\phi_{p}(t)=e^{-i \omega_{p} t} /\left(2 \omega_{p}\right)^{1 / 2}
$$

is constant (and conventionally taken to be unity).

Had an odd term like $\gamma \partial_{t}$ been present in eq. (2.3) the constancy of the Wronskian would have been lost. Hence the possibility of realizing the ETC (2.4) with the help of eq. (2.6) would have been lost as well. This already indicates that, unlike dispersive (real) effects, dissipative effects require more general settings than the above.

\subsection{Interacting models breaking $L I$, general properties}

We now introduce additional degrees of freedom, here after collectively named $\Psi$, which induce dissipation above the energy $\Lambda_{L V}$. We shall work with a particular class of simple models in order to get an exact (non-perturbative) expression for the two-point function of $\phi$ of eq. (1.1). Before introducing these models, we derive general results valid for all unitary QFT's possessing dissipative effects above $\Lambda_{L V}$ in the ground state (the interacting vacuum).

We assume that the total action decomposes as

$$
S_{T}=S_{\phi}+S_{\Psi}+S_{\phi, \Psi}
$$

where the first action is that of eq. (2.1), the second one governs the evolution of the $\Psi$ fields, and the last one the coupling between $\phi$ and these new fields.

We also impose that the last two actions (and the density matrix $\rho_{T}$ ) preserve the homogeneity and isotropy of Minkowski space. From now on, the Cartesian coordinates $t, \mathbf{x}$ are at rest with respect to the preferred frame which is defined by the action $S_{\Psi}+S_{\phi, \Psi}$, i.e. in a covariant notation $\partial_{t} \equiv l^{\mu} \partial_{\mu}$. For these models, the Fourier transform of the Wightman function of eq. (1.1) is of the form

$$
\begin{aligned}
G_{\mathbf{p}, \mathbf{p}^{\prime}}\left(t, t^{\prime}\right) & =\operatorname{Tr}\left[\hat{\rho}_{T} \hat{\phi}_{\mathbf{p}}(t) \hat{\phi}_{\mathbf{p}^{\prime}}^{\dagger}\left(t^{\prime}\right)\right], \\
& =G_{W}\left(t, t^{\prime} ; p\right) \delta^{3}\left(\mathbf{p}-\mathbf{p}^{\prime}\right) .
\end{aligned}
$$

At this point, an important remark should be made. In the presence of interactions, the notion (and the usefulness) of the time-dependent modes given in eq. (2.7) disappears. Instead, the timedependent function $G_{W}\left(t, t^{\prime} ; p\right)$ of eq. (2.9) is always well-defined, for all choices of $\Psi$ and for all actions $S_{\Psi}+S_{\phi, \Psi}$.

When the situation is stationary, $G_{W}\left(p ; t, t^{\prime}\right)$ further simplifies in the frequency representation:

$$
G_{W}\left(t, t^{\prime} ; p\right)=\int \frac{d \omega}{2 \pi} e^{-i \omega\left(t-t^{\prime}\right)} G_{W}(\omega, p) .
$$

To compute $G_{W}(\omega, p)$ in an arbitrary (stationary) state, it is appropriate, as we shall see, to split the analysis by considering separately the commutator $G_{c}$ of $\phi_{\mathbf{p}}$, which is odd in $\omega$, and the anticommutator $G_{a}$, which is even.

When working in the ground state, one reaches the simplest case in the following sense. Whatever the $\Psi$ fields may be, the Fourier transform of the time ordered (Feynman) propagator,

$$
2 G_{F}=G_{a}+G_{c} \operatorname{sign}\left(t-t^{\prime}\right)
$$


is always of the form given in eq. (1.2), and therefore characterized by a single function $\Sigma_{F}(\omega, p)$. When restricting attention to Gaussian models, $\Sigma_{F}(\omega, p)$ is given by a 1-loop calculation, whereas it contains a series of 1PI graphs for non-Gaussian models. In non-vacuum states and in nonstationary situations, $2 \mathrm{pt}$ functions have a more complicated structure. It is to understand the meaning of this strucure that a separate analysis of $G_{c}$ and $G_{a}$ is appropriate.

Let us conclude with two remarks. First, the effective dispersion relation of $\phi$ is a posteriori defined by the poles of eq. (1.2). In this way, non-trivial dispersion relations arise from dynamical processes rather than from being introduced from the outset. The present work therefore provides physical foundations (and restrictions, as later discussed) to the kinematical approach which has been adopted in the literature. Second, from analyzing dynamical models, we shall see that, even in the vacuum, on-shell dissipative effects (i.e. dissipation arising along the minima of the denominator of eq. (1.2) ) are unavoidable when $L I$ is broken in the UV by the action $S_{\Psi}+S_{\phi, \Psi}$, in complete opposition with the fact that on-shell dissipation is forbidden when working (in the vacuum) with $L I$ actions.

\subsection{Gaussian models}

To simplify the calculation of $\Sigma(\omega, p)$ and to get exact non perturbative expressions (in stationary situations), we assume that the action $S_{T}$ is quadratic in all field variables. This is of course a very restrictive hypothesis. However, it should be recalled that we are not after computing $\Sigma$ from first principles. Rather our aim is to determine the modifications of the power spectra, see eqs. (1.6), when including dissipative effects in the UV sector. To this end, it is sufficient to understand how the properties of $\Sigma$ affect the 2 pt function of $\phi$.

Given that we are preserving the homogeneity of Minkowski space, the Gaussian assumption implies that the total action splits as

$$
S_{T}=\int d^{3} p S_{T}(\mathbf{p})
$$

where each action $S_{T}(\mathbf{p})$ depends only on the complex field operator $\phi_{\mathbf{p}}$ and the $\mathbf{p}$-th Fourier component of $\Psi_{i}$ (each of these is a two-mode $(\mathbf{p},-\mathbf{p})$ system). The structure of $S_{T}(\mathbf{p})$ is

$$
\begin{aligned}
S_{T}(\mathbf{p})= & \frac{1}{2} \int d t \phi_{\mathbf{p}}^{*}\left(-\partial_{t}^{2}-\omega_{p}^{2}\right) \phi_{\mathbf{p}}+\frac{1}{2} \Sigma_{i} \int d t \Psi_{i}^{*}(\mathbf{p})\left(-\partial_{t}^{2}-\Omega_{i}^{2}(p)\right) \Psi_{i}(\mathbf{p}) \\
& +\Sigma_{i} \int d t g_{i}(p) \phi_{\mathbf{p}} \Psi_{i}^{*}(\mathbf{p})
\end{aligned}
$$

where $i$ is a discrete (or continuous) index, where $\Omega_{i}(p)$ is the energy of the quanta of the oscillators $\Psi_{i}(\mathbf{p})$, and where $g_{i}(p)$ is the coupling constant at fixed $p, i$.

These Gaussian models have been used since many years (at least since the early 50's) and for different purposes. They have been introduced (in their continuous version) to study nonpertubatively atomic transitions, see [20] and refs. therein, and to model quantum electrodynamics in the dipole approximation, see also [21, 22] for an application to the Unruh effect. They have been used in Quantum Optics and to model quantum Brownian motion [23]. They have also been 
used to study decoherence effects [24]. ${ }^{4}$ Needless to say that our intention is not to provide a new approach to these models, but rather to exploit them to reach our aim: provide unitary models allowing to study dissipative effects in arbitrary curved backgrounds.

Therefore, instead of chosing a priori a specific model, we shall solve the equations of motion without specifying the set of $\Psi_{i}(\mathbf{p})$, their energy $\Omega_{i}(p)$ and their coupling $g_{i}(p)$. We shall choose them in the next subsection to further simplify the equations we shall need to solve. To preserve stationarity in Minkowski space, the $\Omega$ 's and the $g$ 's must be time independent. When $\Omega_{i}^{2}(p) \neq$ $M_{i}^{2}+p^{2}$, the kinetic action of $\Psi_{i}(\mathbf{p})$ breaks $L I$ and defines the preferred frame. On the contrary when $\Omega_{i}^{2}(p)=M_{i}^{2}+p^{2}$ the preferred frame is only defined by the interaction term $S_{\phi \Psi}$ through the $p$-dependence of the coupling functions $g_{i}(p)$.

The equations of motion are

$$
\begin{aligned}
& \left(\partial_{t}^{2}+\omega_{p}^{2}\right) \phi_{\mathbf{p}}=\Sigma_{i} g_{i}(p) \Psi_{i}(\mathbf{p}), \\
& \left(\partial_{t}^{2}+\Omega_{i}^{2}\right) \Psi_{i}(\mathbf{p})=g_{i}(p) \phi_{\mathbf{p}} .
\end{aligned}
$$

The general solution of the second equation reads

$$
\Psi_{i}(\mathbf{p}, t)=\Psi_{i}^{o}(\mathbf{p}, t)+\int d t^{\prime} R_{i}^{o}\left(t, t^{\prime} ; p\right) g_{i}\left(t^{\prime} ; p\right) \phi_{\mathbf{p}}\left(t^{\prime}\right),
$$

where $\Psi_{i}^{o}(\mathbf{p}, t)$ is a free solution which depends on initial conditions imposed on $\Psi_{i}(\mathbf{p})$. The second term contains $R_{i}^{o}\left(t, t^{\prime} ; p\right)$, the (free) retarded Green function of $\Psi_{i}(\mathbf{p})$. It obeys

$$
\left(\partial_{t}^{2}+\Omega_{i}^{2}(p)\right) R_{i}^{o}\left(t, t^{\prime} ; p\right)=\delta\left(t-t^{\prime}\right)
$$

and vanishes for $t<t^{\prime}$. (To prepare the application to time dependent geometries, we have treated $\omega_{p}^{2}, \Omega_{i}^{2}$ and $g_{i}$ as arbitrary functions of time (in cosmology these become indeed time dependent through their dependence in the scale factor $a(t)$ ). ) Injecting eq. (2.16) in eq. (2.14) one gets

$$
\left(\partial_{t}^{2}+\omega_{p}^{2}\right) \phi_{\mathbf{p}}=\Sigma_{i} g_{i}(t ; p) \Psi_{i}^{o}(\mathbf{p}, t)+\Sigma_{i} g_{i}(t ; p) \int d t^{\prime} R_{i}^{o}\left(t, t^{\prime} ; p\right) g_{i}\left(t^{\prime} ; p\right) \phi_{\mathbf{p}}\left(t^{\prime}\right) .
$$

The (exact) solution of this equation has always the following structure

$$
\phi_{\mathbf{p}}(t)=\phi_{\mathbf{p}}^{d}(t)+\int d t^{\prime} G_{r}\left(t, t^{\prime} ; p\right)\left[\Sigma_{i} g_{i}\left(t^{\prime} ; p\right) \Psi_{i}^{o}\left(\mathbf{p}, t^{\prime}\right)\right] .
$$

The first term is the "decaying" solution. It contains all the information about the initial condition of $\phi_{\mathbf{p}}$, and obeys the non local equation

$$
\int d t_{1}\left[\delta\left(t-t_{1}\right)\left(\partial_{t_{1}}^{2}+\omega_{p}^{2}\right)-\Sigma_{i} g_{i}(t ; p) R_{i}^{o}\left(t, t_{1} ; p\right) g_{i}\left(t_{1} ; p\right)\right] \phi_{\mathbf{p}}\left(t_{1}\right)=0 .
$$

${ }^{4}$ Depending on the point of view adopted, these models can be solved and analyzed by means of different methods. In what follows we shall adopt the atomic physics point of view and use the simplest approach based on Heisenberg equations of motion. Since we shall work at zero temperature, this approach is also appropriate because quantum aspects dominate over stochastic ones. Even though legitimate, we have not adopted the "open quantum system" point of view for two reasons. First the general methods used in this approach, see [25], somehow hide the simplicity of the model we are dealing with. In fact only but standard Quantum Mechanics is required to solve it. Secondly, we are planning (in a subsequent work) to study the correlations bewteen $\phi$ and $\Psi_{i}$. Therefore we shall treat $\phi$ and $\Psi_{i}$ on equal footing as in 20, 21, 22]. 
The second term is the "driven" solution. It is governed by the initial conditions of $\Psi_{i}^{o}(\mathbf{p})$ and by the (dressed) retarded Green function, the solution of eq. 2.20) with $\delta\left(t-t_{1}\right)$ on the r.h.s. Therefore the evolution of both $\phi^{d}$ and $G_{r}$ fully takes into account, through the non-local term in the above bracket, the back-reaction due to the coupling to the additional degrees of freedom. In Gaussian models, it is quadratic in $g_{i}$. Hence the solutions $\phi^{d}$ and $G_{r}$ are series containing all powers of $g_{i}$. Therefore Gaussian models do give rise to non perturbative effects. Moreover since $g_{i}(t ; p)$ are arbitrary functions of $p$ and $t$, at this point there is no reason to consider non-Gaussian models.

We conclude this subsection with two remarks. First, eq. (2.19) also furnishes the exact solution for the Heisenberg operator $\hat{\phi}_{\mathbf{p}}$ because the equations we solved are all linear. Since we shall work quantum mechanically, it is relevant to study the correlation functions of $\phi_{\mathbf{p}}$. In Appendix A we present their properties.

Second, eq. (2.20) tells us that in general, the coupling to an environment (even a linear one) gives rise to non-local equations of motion. When dealing with stationary situations this does not cause any problem because, as shown in Appendix B, observables can be (algebraically) computed in the frequency representation. However in non stationary situations and in curved space-times, to be able to compute observables it becomes imperative to simplify eq. (2.20). A question we now address.

\subsection{Time dependent settings}

In Appendix $\mathrm{C}$, we provide a class of stationary models characterized by the power of the ratio $p / \Lambda_{L V}$ which specifies how dissipative effects grow with the energy, see eqs. (1.4, 6.1). This class covers the general case and can be used as a template to study the phenomonological consequences of dissipative effects. In addition, after eq. (5.6), it was noticed that the dissipative properties are governed by the product $g^{2}(\omega, p) R^{o}(\omega, p)$. Therefore many theories with different kinetic and coupling terms will give rise to the same (stationary) phenomenology.

In this Section we exploit this freedom to simplify the expressions having in mind the transposition of our model from Minkowski space to curved metrics. Therefore "good" models should possess two-point functions with simple properties when expressed in the terms of time and space, and not only in Fourier components $\omega, p$.

The core of the problem is that, when dissipation is strong, the relevant observable $G_{a}$ (it gives both the Power Spectrum and Hawking distribution) is given by the "driven" term in eq. (4.6), which is a double integral containing a kernel (which can be computed) and the retarded Green function of $\phi$ which is not known. Indeed it is only implicitely defined as a solution of eq. 2.20), which is, in general, non-local. We are thus led to choose the action of $\Psi$ in order for eq. (2.20) to be local. This implies that the retarded Green function of $\Psi$ appearing in this equation be proportional to $\delta\left(t-t^{\prime}\right)$. Notice that this requirement concerns the Green function of the environment and not that of the system we probe. Thus it will not restrict the phenomenology. 
Given this aim, an appropriate class of models is defined by the action

$$
\begin{aligned}
S_{T}^{(n)}(\mathbf{p})= & \frac{1}{2} \int d t \phi_{\mathbf{p}}^{*}\left(-\partial_{t}^{2}-\omega_{p}^{2}\right) \phi_{\mathbf{p}} \\
& +\frac{1}{2} \int d t \int d k \Psi^{*}(\mathbf{p}, k)\left(-\partial_{t}^{2}-\left(\pi \Lambda_{L V} k\right)^{2}\right) \Psi(\mathbf{p}, k) \\
& +g \Lambda_{L V} \int d t \int d k\left(\frac{p}{\Lambda_{L V}}\right)^{n+1} \phi_{\mathbf{p}} \partial_{t} \Psi^{*}(\mathbf{p}, k) .
\end{aligned}
$$

When compared with the action of eq. (2.13), we have replaced the discrete index $i$ by the integral over the dimensionless variable $k$. As recalled in Appendix B, one needs a continuous spectrum of the environment to have proper dissipation, see discussion after eq. (5.6). The continuous variable $k$ can be viewed as a momentum (expressed in units of $\Lambda_{L V}$ ) in an extra flat fifth spatial dimension. The relationship with the Brane World Scenarios of [17, 18] is clear. In the 'atomic' version of this model [21, 22] which has inspired us, the radiation field $\Psi$ is a massless 2 dimensional field propagating in $t$ and in the dimension associated with $k$.

In $S_{\phi \Psi}$ have factorized out a factor of $\Lambda_{L V}$ so that the coupling constant $g$ is dimensionless. We have also introduced an additional time derivative acting on $\Psi$. This choice leads to the above mentioned $\delta\left(t-t^{\prime}\right)$. Indeed, on the one hand, taking this extra derivative, the continuous character of $k$, and the fact that $g$ is independent of $k$, eq. (2.18) becomes

$$
\left(\partial_{t}^{2}+\omega_{p}^{2}\right) \phi_{\mathbf{p}}=g_{n} \partial_{t} \int d k \Psi^{o}(\mathbf{p}, k, t)-g_{n} \partial_{t} \int d t^{\prime} \int d k R^{o}\left(t, t^{\prime} ; k, p\right) \partial_{t^{\prime}}\left(g_{n} \phi_{\mathbf{p}}\left(t^{\prime}\right)\right),
$$

where $g_{n} \equiv g \Lambda_{L V}\left(p / \Lambda_{L V}\right)^{n+1}$. On the other hand, for each 3-momentum $\mathbf{p}$, the Green function of $\Xi=\int d k \Psi(k)$ is that of a massless 2-dimensional free field. In Fourier it is given by $R^{o}(\omega, k)=$ $1 /\left(-(\omega+i \varepsilon)^{2}+\left(\pi \Lambda_{L V} k\right)^{2}\right)$. Hence it obeys

$$
\partial_{t} \mathbf{R}^{o}\left(t, t^{\prime}\right) \equiv \partial_{t} \int \frac{d \omega}{2 \pi} \int d k R^{o}(\omega, k) e^{-i \omega\left(t-t^{\prime}\right)}=\frac{\delta\left(t-t^{\prime}\right)}{\Lambda_{L V}},
$$

which is the required property to simplify eq. (2.22).

When $g_{n}$ is constant, the retarded Green function of $\phi$ associated to eq. (2.22) obeys the following local equation

$$
\left[\partial_{t}^{2}+\frac{g_{n}^{2}}{\Lambda_{L V}} \partial_{t}+\omega_{p}^{2}\right] G_{r}\left(t, t^{\prime}, p\right)=\delta\left(t-t^{\prime}\right),
$$

To make contact with Appendix B and C, let us rewrite this equation in Fourier transform,

$$
\left[-\omega^{2}-i g^{2} \frac{\omega}{\Lambda_{L V}} p^{2}\left(\frac{p}{\Lambda_{L V}}\right)^{2 n}+\omega_{p}^{2}\right] G_{r}(\omega, p)=1 .
$$

We thus see that $\operatorname{Re} \Sigma_{r}=0$ and that $\operatorname{Im} \Sigma_{r}$ is (exactly) given by $g^{2}$ times the expression of eq. (6.1). Thus, even though we have chosen a simple and definite form for $\mathbf{R}^{o}\left(t, t^{\prime}\right)$, the above action does deliver the $n$ dissipative behaviors of Appendix B by choosing the appropriate power of $p$ in the coupling $g_{n}$ appearing in the action $S_{\phi \Psi}$.

When $g$ and $\omega_{p}^{2}$ are arbitrary time-dependent functions, the Fourier analysis looses its power. However, in time dependent settings, our $G_{r}$ still obeys a local equation:

$$
\left[\partial_{t}^{2}+2 \tilde{\gamma}_{n} \partial_{t}+\left[\omega_{p}^{2}+\partial_{t} \tilde{\gamma}_{n}\right]\right] G_{r}\left(t, t^{\prime}\right)=\delta\left(t-t^{\prime}\right),
$$


where the $n$-th decay rate $\tilde{\gamma}_{n}(t)=g^{2}(t) \gamma_{n}$ is now a definite time dependent function. When transposing the model of eq. (2.21) in expanding homogeneous universes, the Green function will therefore also obey a local equation.

\subsection{Covariant description}

We now provide the covariant version of the action of eq. (2.21). This expression will then be used to define our theory in curved backgrounds. ${ }^{5}$

Two steps should be done. We need to go from $\mathbf{p}$ considerations to a local description, and express the various actions in terms of the unit vector field $l^{\mu}$ and the spatial metric $\perp^{\mu v}$ defined in eq. (1.3). Both are straightforward and, in arbitrary coordinates, the total action $S_{T}^{(n)}=\int d^{3} p S_{T}^{(n)}(\mathbf{p})$ reads (in Minkowski space-time)

$$
\begin{aligned}
S_{T}^{(n)}= & -\frac{1}{2} \int d^{4} x \sqrt{-g} g^{\mu v} \partial_{\mu} \phi \partial_{v} \phi \\
& \left.+\frac{1}{2} \int d^{4} x \sqrt{-g} \int d k\left[\left(l^{\mu} l^{v}-c_{\Psi}^{2} \perp^{\mu v}\right) \partial_{\mu} \Psi_{k} \partial_{v} \Psi_{k}-\left(\pi \Lambda_{L V} k\right)^{2} \Psi_{k}^{2}\right)\right] \\
& +g \Lambda_{L V} \int d^{4} x \sqrt{-g}\left(\left(\frac{\Delta}{\Lambda_{L V}^{2}}\right)^{(n+1) / 2} \phi\right) l^{\mu} \partial_{\mu} \int d k \Psi_{k},
\end{aligned}
$$

where $\Delta$ is the Laplacian on the three surfaces orthogonal to $l^{\mu}$.

We have slightly generalized the action of eq. (2.21) by subtracting $c_{\Psi}^{2} \perp^{\mu v}$ to the kinetic term of $\Psi$, where $c_{\Psi}^{2} \ll 1$. With this new term, $p$-th components of the field $\Psi_{k}$ are now massive fields which propagate with a velocity (relative to $l^{\mu}$ ) whose square is bounded by $c_{\Psi}^{2}$. In addition they now possess a well defined energy-momentum tensor which can be obtained by varying their action with respect to $g^{\mu v}$. To obtain the simplified expressions we use in the paper the (regular) limit $c_{\Psi}^{2} \rightarrow 0$ should be taken.

In this limit, the (free) retarded Green function of the $\Xi=\int d k \Psi$ field obeys a particularly simple equation when expressed in space-time coordinates. Indeed, it obeys

$$
l^{\mu} \frac{\partial}{\partial x^{\mu}} \int d k R^{o}(x, y ; k) \equiv l^{\mu} \frac{\partial}{\partial x^{\mu}} \mathbf{R}^{o}(x, y)=\frac{1}{\Lambda_{L V}} \frac{\delta^{4}\left(x^{\mu}-y^{\mu}\right)}{\sqrt{-g}},
$$

\footnotetext{
${ }^{5}$ Perhaps the motivations for these two steps require further explanation since another referee wrote: "I do not see the point of a covariant description of the $\Psi$ field since it is nothing more than a convenient parametrization of the environment degrees of freedom." Several points should be mentionned. First, we are after describing vacuum effects when Lorentz Invariance is broken in the UV. Therefore the breakdown of LI must come locally [19]. Second, as in studies of dispersive effects, we require that our QFT be well defined in an arbitrary background. It would be physically questionable (or even meaningless) to study dissipative effects on the observables of eq. (1.6) from theories which are only defined in a particular class of space-times (e.g. homogeneous spaces). Third, we have introduced the $\Psi$ field to characterize the effects of dissipation which would stem from fundamental theories obeying the Equivalence Principle, see 17] for a prototype. Fourth, from the point of view of open quantum systems, it could a priori seem inappropriate to proceed to a covariantization, since in most situations (heat bath, condensed matter systems) there is a preferred frame which is globally defined. However, when the system is non-homogeneous (e.g. a fluid characterized by a nonhomogeneous flow), low energy fluctuations effectively live in a "acoustic" curved geometry [27]. Moreover, in this case, short distance effects, i.e. dispersive (or dissipative) effects, are covariantly described when using this metric [12, 19, because they arise locally. In brief, we shall write down actions in curved space using the EP which requires a covariant description.
} 
On the r.h.s, one finds the "invariant" delta function with respect to the invariant measure $d^{4} x \sqrt{-g}$. This equation is nothing but the covariantized and "localized" version of eq. (2.23). Its physical meaning is clear. It tells us that the back-reaction of $\phi(x)$ onto itself through $\Xi$ will be local at every point in space-time.

The equations of motions do not have a particularly simple form when expressed in space-time coordinates. Using the condensed notation $\Xi=\int d k \Psi$, the relevant equations read

$$
\frac{1}{\sqrt{-g}} \partial_{\mu} \sqrt{-g} g^{\mu v} \partial_{\nu} \phi(x)=g \Lambda_{L V} \frac{1}{\sqrt{-g}}\left(\frac{\Delta}{\Lambda_{L V}^{2}}\right)^{\frac{n+1}{2}} \sqrt{-g} l^{\mu} \partial_{\mu} \Xi(x),
$$

where the interacting field $\Xi$ field is

$$
\Xi(x)=\Xi^{o}(x)-g \Lambda_{L V} \int d^{4} y \sqrt{-g} \mathbf{R}^{o}(x, y)\left[\frac{1}{\sqrt{-g}} \partial_{\mu}\left(l^{\mu} \sqrt{-g}\left(\frac{\Delta}{\Lambda_{L V}^{2}}\right)^{\frac{n+1}{2}} \phi(y)\right)\right] .
$$

When inserting eq. (2.30) in eq. (2.29), using eq. (2.28), one verifies that the dissipative term is local and first order in $l^{\mu} \partial_{\mu}$. As wished, dissipation occurs along the preferred direction.

\section{Dissipative effects in curved background geometries}

\subsection{The Lagrangian and the properties of $\Psi$}

To define a dissipative QFT in an arbitrary curved geometry, we need some principles. From a physical point of view, we adopt the Equivalence Principle, or better what can be considered as its generalization in the presence of the additional unit time-like vector field. We are in fact dealing with two (sets of) dynamical fields, the $\phi$ field we probe, and the $\Psi_{i}$ fields we do not; but also with two background fields $g_{\mu \nu}$ and $l^{\mu}$. The Generalized Equivalence Principle means that the action densities of the dynamical fields be given by scalar functions (under general coordinate transformations) which reduce to those one had in Minkowski space time and for a homogeneous and static $l^{\mu}$ field, i.e. those of eq. (2.27).

The densities are thus not completely fixed by the GEP, as it was the case with the EP. For a scalar field in a curved geometry, there was always the possibility of considering a non-minimal coupling to gravity by adding to the lagrangian a term proportional to $R \phi^{2}$. In the present case, the ambiguity is larger because the vector $l^{\mu}$ allows to form new scalars, such as its expansion

$$
\Theta \equiv \nabla_{\mu} l^{\mu}=(-g)^{-1 / 2} \partial_{\mu}\left[(-g)^{1 / 2} l^{\mu}\right],
$$

where $\nabla_{\mu}$ is the covariant derivative with respect to the metric $g_{\mu v}$. The ambiguity can only be resolved by adopting some additional principle, such as the principle of minimal couplings which forbids adding such scalars.

Rather than adopting it, our second principle (which simplifies the equations of motion in curved backgrounds, but which is by no means necessary) is that the locality of the back-reaction effects of $\phi$ through $\Xi$ be preserved. That is, we choose the non-minimal coupling of $\Xi$ so as to keep eq. (2.28). As shown later, this will also guarantee that the proper frequency of the $\Psi_{k}$ fields measured along $l^{\mu}$ stays constant as the universe expands, and more generally, in any curved background. Starting from eq. (2.27), the locality is preserved by replacing both in $S_{\Psi}$ and $S_{\Psi \phi}$

$$
l^{\mu} \partial_{\mu} \Psi_{k} \rightarrow \mathscr{D}_{l} \Psi_{k} \equiv l^{\mu} \partial_{\mu} \Psi_{k}+\frac{\Theta}{2} \Psi_{k}=\frac{1}{2}\left(l^{\mu} \nabla_{\mu} \Psi_{k}+\nabla_{\mu}\left[l^{\mu} \Psi_{k}\right]\right)
$$


where $\partial_{\mu}$ is the partial derivative.

To simplify the forthcoming equations, we use the fact that one can always work in "preferred" coordinate systems in which the shift $l^{i}$ vanishes and in which the preferred time is such that $l_{0}=1$. (We assume that the set of orbits of $l^{\mu}$ is complete and without caustic. In this case, every point of the manifold is reached by one orbit.) It will be found useful to work in these coordinate systems with rescaled fields $\Psi^{r} \equiv(-g)^{1 / 4} \Psi$ because one can then group the above two terms into a single expression:

$$
\mathscr{D}_{l} \Psi_{k}=(-g)^{-1 / 4} l^{\mu} \partial_{\mu}\left((-g)^{1 / 4} \Psi_{k}\right)=(-g)^{-1 / 4} l^{\mu} \partial_{\mu} \Psi_{k}^{r} .
$$

Notice also that there exists a subclass of background fields $(g, l)$, for which one can find coordinate systems such that both the shift $l^{i}$ and $g^{o i}$ vanish. In these comoving coordinate systems, the above equations further simplify since $-g=h_{c}$ where $h \equiv \operatorname{det}\left(\perp_{i j}\right){ }^{6}$

Having chosen this non-minimal coupling, one verifies that the kinetic term of the rescaled fields $\Psi_{r}$ is insensitive to the "curvature" of both $g_{\mu \nu}$ and $l^{\mu}$ (when the limit $c_{\Psi}^{2} \rightarrow 0$ is taken). Moreover the differential operator which acts on the retarded Green function of $\Psi_{r}$ in the equation of motion of $\phi$, see eqs. (2.29, 2.30), is also "flat" thereby guaranteeing that the modified version eq. (2.28) still applies, that is

$$
\begin{aligned}
\mathscr{D}_{l} \mathbf{R}^{o}(x, y) & =(-g(x))^{-1 / 4} l^{\mu} \partial_{\mu}\left(\mathbf{R}_{r}^{o}(x, y)\right)(-g(y))^{-1 / 4} \\
& =\frac{1}{\Lambda_{L V}} \frac{\delta^{4}\left(x^{\mu}-y^{\mu}\right)}{\sqrt{-g}}
\end{aligned}
$$

where $\mathbf{R}_{r}^{o}(x, y)$ is the retarded Green function of the rescaled field $\Psi_{r}$. It obeys (in preferred coordinate systems) $\partial_{t} \mathbf{R}_{r}^{o}=\delta^{4} / \Lambda_{L V}$, and "defines" the retarded Green function $\mathbf{R}^{o}=(-g)^{1 / 4} \mathbf{R}_{r}^{o}(-g)^{1 / 4}$ which is a bi-scalar. Hence the equations of motion in an arbitrary background "tensor-vector metric" specified by the couple $\left(g_{\mu \nu}, l^{\mu}\right)$, are given by eqs. (2.29, 2.30, 3.4 with the substitution of eq. (3.2).

Several remarks should be made. First, from the simplified equation $\partial_{t} \mathbf{R}_{r}^{o}=\delta^{4} / \Lambda_{L V}$ it might seem that the background tensor metric $g_{\mu \nu}$ plays no role. This is not true, it enters indirectly as it is used to normalize the vector field $l^{\mu}$ at every point. Therefore, it intervenes in the specification that the (proper) energy scale $\Lambda_{L V}$ stays fixed as the universe (or the comoving volume $h_{c}$ ) expands.

Second, in the limit $c_{\Psi}^{2} \rightarrow 0$, the (rescaled) $\Psi_{k}$ fields define a new kind of field. They propagate in an effective space-time given by the time development of the 3-dimensional set of orbits of the $l^{\mu}$ field. Indeed, at fixed $k, \Psi_{k}(x)$ can be decomposed in non-interacting local field-oscillators, each of them evolving separately along its orbit. This situation is similar to the long wave length (gradient-free) expression of the action of Steward and Salopek[33]. In the absence of $l^{\mu}$, the space time geometry must be (nearly) homogeneous for the action of the modes to possess this decomposition. However, when the $l^{\mu}$ field is given, one can identify, even in non-homogeneous

\footnotetext{
${ }^{6}$ To illustrate this point, let us consider FLRW flat metrics. In comoving coordinates, one has $d s^{2}=-d t^{2}+a^{2} d x^{2}$, and $h=h_{c}=a^{6}$. In Lemaitre coordinates $X=a x$, one has $d s^{2}=-d t^{2}+(d X-V d t)^{2}$, where the velocity is $V=H X$. The spatial sections are now the Euclidean space with $h=1$, and the (contravariant) components of the unit vector field are $l^{\mu}=1, V$. To compute $h_{c}$ one should solve the equation of motion of comoving (free falling) observers $d X-V d t=0$, and use the initial position as new coordinates. This procedure will be done in subsection 3.3 starting with PainlevéGullstrand coordinates to describe the black hole metric and $l^{\mu}$ field.
} 
metrics, every point in space-time in an invariant way by the spatial position of the corresponding "preferred" orbit at some time, and the proper time along the orbit (as long as $l^{\mu}$ has no caustic). We can thus build covariant actions exploiting this possibility and consider fields composed of a dense set of local oscillators (i.e. one has 1 degree of freedom per point) at rest with respect to $l^{\mu}$. The fields $\Psi_{k}$ belong to this class of fields here after called Chelva fields.

Third, the analogy between the above formalism and the DeWitt [26] way to handle the short distance behavior of Green (Hadamard) functions seems worth deepening.

Fourth, even though we have chosen to simplify the equation of motion of $\phi$ by imposing that the rescaled kernel obeys $\partial_{t} \mathbf{R}_{r}^{o}=\delta^{4} / \Lambda_{L V}$, the 2 pt functions of $\phi$ in curved backgrounds are highly non trivial, and in particular the anti-commutator (which governs both $P_{k}$ and $n_{\omega}$ in eq. (1.6)). The reason is double, first $G_{a}$ will be given by a double time integral, see eq. (4.6), and second it is the kernel $\mathbf{N}$ and not $\mathbf{R}$ which is the source of $G_{a}$.

\subsection{Application to cosmology}

To get the equation of motion we consider eq. (2.27) (with the curved metric modifications introduced in the former subsection) in a FLRW metric $d s^{2}=-d t^{2}+a^{2}(t) d \mathbf{x} d \mathbf{x}$. To simplify the notations, we use the conformal time $d \eta=d t / a$ and work with the rescaled fields $\phi_{r}=a \phi$ and $\Psi_{r}(k)=a^{3 / 2} \Psi(k)$. Notice that their power in $a$ differs. Dropping these $r$ indices, working in Fourier transform with respect to the (dimensionless) comoving coordinates $\mathbf{x}$, the equation of motion of Heisenberg operator $\phi_{\mathbf{p}}$ is

$$
\left(\partial_{\eta}^{2}+2 \gamma_{n} \partial_{\eta}+\left(\omega_{p}^{2}(\eta)+\partial_{\eta} \gamma_{n}\right)\right) \phi_{\mathbf{p}}=g_{n} \partial_{\eta} \Xi^{o}(\mathbf{p}),
$$

The conformal frequency $\omega_{p}^{2}(\eta)=p^{2}-\partial_{\eta}^{2} a / a$ is that of a rescaled minimal coupled massless field. In this expression, as everywhere in this subsection, $p$ is the conformal (dimensionless and constant) wave vector. The time dependent coupling coefficient is

$$
g_{n} \equiv g a^{1 / 2} \Lambda_{L V}\left(p / a \Lambda_{L V}\right)^{n+1}
$$

Its unusual $a$-dependence follows from the Generalized Equivalence Principle and by having taken into account the different rescaling of the two fields.

Straightforward algebra gives $\gamma_{n}(\eta)$, the decay rate in conformal time. When compared to the comoving frequency $p$ to get the relative strength of dissipation, one gets

$$
\frac{\gamma_{n}(\eta)}{p}=\frac{1}{2}\left(\frac{p}{a \Lambda_{L V}}\right)^{2 n+1}=\frac{1}{2}\left(\frac{p_{p h y s}(a)}{\Lambda_{L V}}\right)^{2 n+1} .
$$

In the last equality we have re-introduced the proper momentum $p_{\text {phys }}(a)=p / a$. With this equation we verify that, at any time in an expanding universe and for any mode $\phi_{p}$, the relative strength of dissipation is simply that obtained in Minkowski space at the corresponding energy scale, see eq. (6.2). This is nothing but the expression of the GEP, at the level of the dynamical equations.

Notice however that the equation of motion in an expanding universe contains a frequency shift

$$
\frac{g_{n}^{2}}{\Lambda_{L V}} \partial_{\eta}\left(\ln g_{n}\right)=p^{2}\left(\frac{a H}{p}\right)\left(\frac{p}{a \Lambda_{L V}}\right)^{2 n+1} \text {. }
$$


We have factorized out the unperturbed frequency square to get the relative value of the shift. It vanishes both when dissipation is negligible and when $a H / p \ll 1$, i.e. when the expansion rate $H$ is negligible with respect to the proper momentum. From this expression we can already conclude that it cannot play any role when the two relevant scales $H$ and $\Lambda_{L V}$ are well separated, i.e. when

$$
\sigma \equiv \frac{H}{\Lambda_{L V}} \ll 1
$$

Indeed when the physical momentum is high and of the order of $\Lambda_{L V}$, the relative frequency shift is proportional to $\sigma$, and when the physical momentum is of the order of $H$ (at horizon exit, see the Figure), it is proportional to $\sigma^{2 n+1}$.

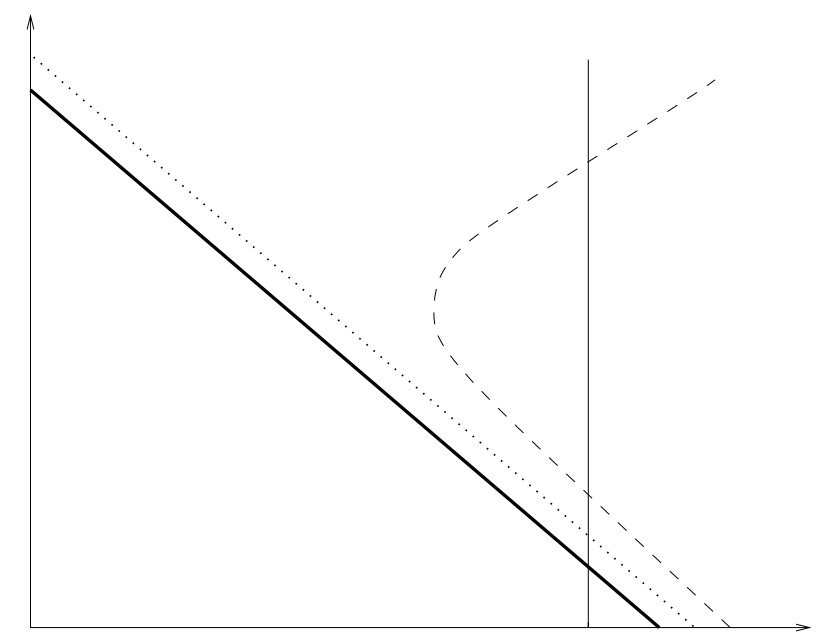

Figure caption. We have represented in a log-log plot and by a dashed line the evolution of $d_{H}=R_{H} / a$, the Hubble radius in comoving coordinates, as a function of a, both during inflation wherein $d_{H} \propto 1 / a$ and during the radiation era wherein $d_{H} \propto a$. We have represented by $a$ thick line the trajectory of the cutoff length scale $d_{\Lambda}=1 / a \Lambda_{L V}$ in the case where $1 / \Lambda_{L V} \ll R_{H}$ during inflation. The dotted line corresponds to an intermediate fixed proper length $\lambda$ which obeys $1 / \Lambda_{L V} \ll \lambda \ll R_{H}^{\text {infl. }}$. The vertical line represents a fixed comoving scale $d_{p}=1 / p$. Below the cutoff length, all modes are overdamped. When a mode exits the cutoff length, it becomes underdamped and starts propagating. When it crosses the intermediate length $\lambda$, it behaves as a free mode, and gets amplified only when exiting the Hubble radius. As explained in the text, adiabaticity, which is guaranteed by $1 / \Lambda_{L V} \ll R_{H}$, guarantees in turn that, near $\lambda$, modes are all born in the BunchDavies vacuum when the environment $\Psi$ is in its ground state.

In quantum settings however, it is not sufficient that the equations of motion possess their Minkowskian form because the quantum state of the system might be affected by the combined effect of dissipative effects and the expansion rate.

In free settings, when assuming that all $\phi_{\mathbf{p}}$ are in their ground state at the onset of inflation, i.e. working in the Bunch-Davies vacuum, the anti-commutator, see eq. (4.5), evaluated at equal time $\eta$ is simply given by

$$
G_{a}^{\text {free }}(\eta, p)=\left|\phi_{\mathbf{p}}^{i n}\right|^{2}=\frac{1}{(2 p)}\left(1+\frac{1}{(p \eta)^{2}}\right)
$$


In the last equality we worked for simplicity with a constant Hubble parameter $H$, in which case $a=-1 / H \eta$. Then the power spectrum of the physical (un-rescaled) field given by

$$
P_{p}^{\text {free }}(\eta)=2 p^{3} \frac{G_{a}^{\text {free }}(\eta, p)}{a^{2}(\eta)}=H_{p}^{2}\left(1+(p \eta)^{2}\right)
$$

becomes constant after horizon exit. We have added a $p$ subscript to $H$ because in slow roll inflation, the relevant value of $H(t)$ for the $p$-mode is that evaluated at horizon exit, i.e. $H_{p}=H\left(t_{p}\right)$, where $t_{p}$ is given by $p / a=H$. The above equation shows that $P_{p}$ acquieres some scale dependence through $H_{p}$. Similarly the deviations from this standard behavior stemming from some UV modification of the theory will also depend on $p$ through $H_{p}$ (and its derivatives). For an explicit example, we refer to [32] where the modifications of the spectrum stem from the fact that $p \eta_{\text {in }}$ is taken large but finite.

In the presence of dissipation, the expression for $G_{a}$ radically differs from the above. Indeed, when dissipative effects grow with the energy (as we suppose it is the case), one reaches the conclusion that in inflation the decaying solution of eq. (3.5) is completely erased (unless one fine-tunes the number of e-foldings so as to keep a residual amplitude). That is, the mode operator is entirely given by its driven term, the second term in eq. (2.19). Hence the power spectrum is also purely driven and given by the second term of eq. (4.6):

$$
G_{a}^{d r i v e n}(\eta, \eta, p)=\int d \eta_{1} \int d \eta_{2} G_{r}\left(\eta, \eta_{1}, p\right) G_{r}\left(\eta, \eta_{2}, p\right) \mathbf{N}\left(\eta_{1}, \eta_{2}, p\right),
$$

where $G_{r}$ is the retarded Green function, solution of eq. (3.5) with $\delta\left(\eta-\eta_{1}\right)$ as a source, and where the kernel $\mathbf{N}$ is the anti-commutator of $g_{n} l^{\mu} \partial_{\mu} \Psi_{p}$, the source of eq. (3.5). From eq. (3.12) we learn that only the (initial) quantum state of the environment matters. In other words, because of the strong dissipation at early times, the power spectrum is independent of the initial state of $\phi$, whatever it was.

In spite of these differences, one can show that when the two scales are well separated, when eq. (3.9) is satisfied, and when the environment is in its ground state, the predictions are unchanged, i.e. the power spectrum obtained from $G_{a}^{\text {driven }}$ coincides with that obtained with $G_{a}^{\text {free }}$ because the combined evolution of $\phi+\Psi$ consists in a parametric (adiabatic) succession of stationary states ordered by the scale factor $a$.

The proof goes in two steps. First scale separation, guarantees the adiabaticity of the evolution. That is, when $\eta$ and $\eta^{\prime}$ are close (in the sense that $1-a(\eta) / a\left(\eta^{\prime}\right) \ll 1$ ), $G_{a}^{\text {driven }}$ is well approximated by

$$
G_{a}^{\text {driven }}\left(\eta, \eta^{\prime}, p\right) \simeq G_{a}^{\text {statio }}\left(\eta-\eta^{\prime} ; \omega_{p}(a), g_{p}(a)\right)=\int \frac{d \omega}{2 \pi} e^{i \omega\left(\eta-\eta^{\prime}\right)} G_{a}\left(\omega ; \omega_{p}(a), g_{p}(a)\right),
$$

where $G_{a}\left(\omega ; \omega_{p}(a), g_{p}(a)\right)$ is the Fourier $\omega$-component of the anti-commutator calculated with the Hamiltonian characterized by the constant values of the frequency $\omega_{p}(a)$ and the coupling $g_{p}(a)$ both evaluated with $a=a(\eta)$. In Appendix $\mathrm{B}$, the stationary value of these anti-commutators has been algebraically solved for all frequencies and all couplings, see eq. (5.11). Moreover, since by hypothesis, we are in the vacuum, eq. (5.9) also applies. In other words, the value of the anti-commutator follows from that of $G_{c}$. This is enough as it guarantees that when the 
mode $\phi_{p}$ becomes free, i.e. much after $\Lambda_{L V}$-exit but before horizon-exit $\left(H \ll p / a \ll \Lambda_{L V}\right)$, eq. (5.14) applies, see the Figure. Thus, irrespectively of what was the coupling with the environment, adiabaticity implies

$$
G_{a}^{\text {driven }}(\eta, \eta, p) \rightarrow G_{a}^{\text {free }}(\eta, \eta, p)=\frac{1}{2 \omega_{p}(a)},
$$

thereby guaranteeing that no modification of the Power Spectrum will be found. In brief adiabaticity means that the evolution proceeds slow enough for not inducing any (non-adiabatic) transition which in the present case would correspond to pair creation.

The second part of the proof consists in providing an upper bound for the probability amplitude of obtaining a non-adiabatic transition. This amplitude is governed by the relative frequency change

$$
\frac{\partial_{\eta} \omega_{p}^{\text {eff }}}{\left(\omega_{p}^{\text {eff }}\right)^{2}}=-\frac{\gamma_{n}^{2} \partial_{\eta} \ln \gamma_{n}}{\left(p^{2}-\gamma_{n}^{2}\right)^{3 / 2}}=(2 n+1) \frac{H \gamma_{\text {phys }}^{2}}{\left(p_{\text {phys }}^{2}-\gamma_{p h y s}^{2}\right)^{3 / 2}},
$$

where $p_{\text {phys }}=p / a$ and $\gamma_{\text {phys }}=\gamma / a$ are the physical momentum and decay rate. Therefore, going backwards in time from the free regime in the underdamped regime, up to $p_{\text {phys }}^{2}>4 \gamma_{\text {phys }}^{2}$ (i.e. $p_{\text {phys }}<\Lambda_{L V}$ ), the non adiabatic parameter raises from zero but stays bounded by

$$
\frac{\partial_{\eta} \omega_{p}^{e f f}}{\left(\omega_{p}^{e f f}\right)^{2}}<3(2 n+1) \sigma\left(\frac{p_{\text {phys }}}{\Lambda_{L V}}\right)^{4 n+1}<3(2 n+1) \sigma \ll 1 .
$$

This guarantees that the amplitude for the system to jump out the ground state is bounded by $\sigma$ (up to an overall factor which plays no role). There is no need to study the stability of the ground state in the transitory regime from underdamped to the overdamped modes (for $p_{\text {phys }}>\Lambda_{L V}$ ), because whatever transitions happened is suppressed by a factor $e^{-\int \gamma d t} \simeq \exp (-1 / \sigma(2 n+1)) \ll 1$. This completes the proof that scale separation guarantees adiabaticity.

\subsection{Dissipation in Black Hole metric}

In spite of the fact that the background metric is no longer homogeneous, the effects of dissipation on Hawking radiation can be studied along lines similar to the above.

For simplicity we consider only spherically symmetric and stationary BH metrics. We also choose the unit vector field $l^{\mu}$ to be stationary and associated with Freely Falling observers which start at rest at infinity. In this case, the expressions for both the metric and $l^{\mu}$ simplify using the $P G$ (Painlevé-Gullstrand) coordinates, $t, r, \theta, \phi$. One has

$$
\begin{aligned}
d s^{2} & =-d t^{2}+(d r-v d t)^{2}+r^{2} d \Omega^{2}, \\
l_{\mu} & =(1, v(r), 0,0), \\
g_{\mu \nu} & =-l_{\mu} l_{v}+\perp_{\mu v}, \quad \text { with } \perp_{\mu v}=\operatorname{diag}\left(0,1, r^{2}, r^{2} \sin ^{2} \theta\right),
\end{aligned}
$$

where $v(r)<0$ is the radial velocity of the FF observers, and $t$ their proper time, not to be confused with the "Schwarzschild" (Killing) time. From the last equation we learn that the 3-surfaces perpendicular to $l^{\mu}$ are simply the Euclidean space. Therefore $h=\operatorname{det} \perp_{i j}=r^{4} \sin ^{2} \theta$ is independent of the expansion of $v$, as it is the case in cosmology in Lemaitre coordinates, see footnote 6 . 
However $P G$ coordinates are not comoving along the $l^{\mu}$ field since the shift is given by $\vec{v}$. To determine the space-time dependence of the comoving volume element $\sqrt{h_{c}}$, we introduce the "preferred" coordinate $r_{0}=r_{0}(t, r)$ defined by

$$
\int_{r_{0}}^{r} \frac{d r^{\prime}}{v\left(r^{\prime}\right)}=t
$$

By definition $r=r\left(t, r_{0}\right)$ gives the trajectory of the FF observer who started from $r_{0}$ at $t=0$. Thus, for every $(t, r), r_{0}=r_{0}(t, r)$ gives the value of radial coordinate at time $t=0$. Using

$$
d r-v d t=\frac{\partial r}{\partial r_{0}} d r_{0}=\frac{v(r)}{v\left(r_{0}\right)} d r_{0}
$$

the line element in preferred (and comoving) coordinates $\left(t, r_{0}\right)$ reads

$$
d s^{2}=-d t^{2}+\left(\frac{v(r)}{v\left(r_{0}\right)}\right)^{2} d r_{0}^{2}+r^{2} d \Omega^{2}
$$

where $r \equiv r\left(t, r_{0}\right)$. Therefore, the evolution of a comoving volume centered along the $r_{0}$-FFtrajectory is given by

$$
h_{c}^{1 / 2}\left(t, r_{0}\right)=\frac{v(r)}{v\left(r_{0}\right)} \times r^{2} \sin \theta=h_{c 2 D}^{1 / 2} \times r^{2} \sin \theta
$$

As in cosmology, it is a well-defined time dependent function. In the present case, it is governed by the (shift) function $v(r)$, and the FF trajectories $r=r\left(t, r_{0}\right)$.

Because of spherical symmetry, our Gaussian action separates into actions at fixed angular momentum : $S_{T}=\Sigma_{l, m} S_{T}(l, m)$, see eq. (2.12). Each of them contains two-dimensional fields $\phi_{l, m}(t, r)$ and $\Psi_{l, m}(t, r)$. We here consider only the s-wave sector, and drop the 0,0 index. We work with the rescaled field $\tilde{\phi}=r \phi, \tilde{\Psi}=r \Psi$ and drop the tilde. Taking into account the 4D character of the problem and eqs. (3.2, 3.3), the action reads

$$
\begin{aligned}
S_{T}= & \frac{1}{2} \iint d t d r r^{2}\left[\left(\left(\partial_{t}+v \partial_{r}\right) \frac{\phi}{r}\right)^{2}-\left(\partial_{r} \frac{\phi}{r}\right)^{2}\right] \\
& +\frac{1}{2} \int d k \iint d t d r r^{2}\left[\left(\left(\partial_{t}+\frac{v \partial_{r}+\partial_{r} v}{2}\right) \frac{\Psi_{k}}{r}-\left(\left(k \Lambda_{L V} \pi\right) \frac{\Psi_{k}}{r}\right)^{2}\right]\right. \\
& +g \Lambda_{L V} \iint d t d r r^{2}\left[\left(\left(\frac{\partial_{r}}{\Lambda_{L V}}\right)^{n+1} \frac{\phi}{r}\right)\left(\left(\partial_{t}+\frac{v \partial_{r}+\partial_{r} v}{2}\right) \int d k \frac{\Psi_{k}}{r}\right)\right] .
\end{aligned}
$$

By varying this action, there is no difficulty to get the equations of motion. One verifies in particular that the kinetic action of the (rescaled) $\Psi^{r}=h_{c 2 D}^{1 / 4} \tilde{\Psi}$ field is as in 2D flat space when expressed in preferred coordinates $t, r_{0}$ :

$$
S_{\Psi}=\frac{1}{2} \int d k \iint d t d r_{0}\left[\left(\partial_{t} \Psi_{k}^{r}\right)^{2}-\left(\left(k \Lambda_{L V} \pi\right) \Psi_{k}^{r}\right)^{2}\right]
$$

This means that the Chelva field $\Psi_{k}^{r}(t, r)$ is a collection of independent oscillators labelled by $r_{0}$. In other words, $\Psi_{k}^{r}(t, r)$ depends on $t$ and $r$ as

$$
\Psi_{k}^{r}(t, r)=\Psi_{k}^{r}\left(t ; r_{0}(t, r)\right) .
$$


One also verifies $l^{\mu} \partial_{\mu} \Psi_{k}^{r}(t, r)=\left.\partial_{t} \Psi_{k}^{r}\left(t ; r_{0}\right)\right|_{r_{0}}$, because $l^{\mu} \partial_{\mu} r_{0}(t, r)=0$, by definition. These facts guarantee that eq. (3.4) applies.

By a direct analysis of the equation of motion, one obtains two important properties. First, one verifies that there is no "dilution" of the dissipation rate as time passes. This is non trivial (see [30] for an a priori similar system in which a dilution does occur). This steady behavior is due to the fact that the Chelva fields form a 3 dimensional dense set (as opposed to the discrete lattice of [30]) which allows the various $h_{c}$ to cancel each other in the determination of the dissipation rate. Moreover one also verifies that stationarity is preserved, not only in the dissipative aspects, but also in the driven properties. This is non trivial either since $h_{c}$ depends on $t$ and appears in the action $S_{\Psi \phi}$ when working with the rescaled fields $\Psi^{r}$.

With these two results, one can study the impact of dissipation on Hawking radiation by applying the technics of [14] which were further developed in [31] [34] to study the impact of dispersion. When the two scales are well separated, i.e. when $\kappa / \Lambda_{L V} \ll 1$, where $\kappa=\partial_{r} v$ evaluated at the horizon $v=-1$, one can work in the near horizon approximation, wherein $v=-1+\kappa x$, and in the $p$-representation. In this representation, the velocity profile becomes a non-trivial operator $\hat{v}=-1-i \kappa \partial_{p}$, in fact the only one. When working at fixed Killing frequency $\omega_{K}$, one obtains the radial propagation of the interacting degrees of freedom in terms of an adiabatic evolution of Green functions and kernels written in the $p$-representation. These are given in Appendix B, with the frequency $\omega=i \partial_{t}$ of that Appendix replaced by the FF frequency $\Omega=i \partial_{t}+i v \partial_{r}=\omega_{K}-\hat{v} p$. We are planning to report on this soon.

Acknowledgements. I am grateful to Dani Arteaga and Enric Verdaguer for common work allowing me to deepen my understanding of dissipative effects. I am also grateful to Ted Jacobson for having repetitively invited me to covariantize my action. I am thankful to many people for having had the opportunity to present this work starting with the IAP in October 2004. This work has been supported by the European Science Foundation network programme "Quantum Geometry and Quantum Gravity".

\section{Appendix A: Two-point correlation functions}

Since the models we consider are Gaussian, the complex function of eq. (2.9) governs all observables built with the Heisenberg field operator $\phi$. To analyse it, as mentioned in the text, it is appropriate to study separately the commutator and the anti-commutator.

We start with the simple part, the commutator

$$
G_{c}\left(t, t^{\prime} ; p\right) \delta^{3}\left(\mathbf{p}-\mathbf{p}^{\prime}\right) \equiv \operatorname{Tr}\left[\rho_{T}\left[\phi_{\mathbf{p}}(t), \phi_{\mathbf{p}^{\prime}}^{\dagger}\left(t^{\prime}\right)\right]_{-}\right] .
$$

It possesses several (well-known) properties. First, using eq. 2.19) one sees that it decomposes into two terms, one due to the non-commuting character of $\phi^{d}$, the other due to that of $\Psi_{i}^{0}$. Second, since both commutators are c-numbers, it is independent of $\rho_{T}$, the state of the system. Hence, for all Gaussian models, one has

$$
G_{c}\left(t, t^{\prime} ; p\right)=\left[\phi^{d}(t), \phi^{d}\left(t^{\prime}\right)\right]_{-}+\iint d t_{1} d t_{2} G_{r}\left(t, t_{1}\right) G_{r}\left(t^{\prime}, t_{2}\right) D\left(t_{1}, t_{2}\right),
$$


where the "dissipative" kernel $D\left(t_{1}, t_{2}\right)$ is given by

$$
D\left(t_{1}, t_{2}\right)=\Sigma_{i} \Sigma_{j} g_{i}\left(t_{1}\right) g_{j}\left(t_{2}\right)\left[\Psi_{i}^{o}\left(t_{1}\right), \Psi_{j}^{o}\left(t_{2}\right)\right]_{-}=\Sigma_{i} g_{i}\left(t_{1}\right) G_{c, i}^{o}\left(t_{1}, t_{2}\right) g_{i}\left(t_{2}\right) .
$$

Notice how this kernel combines the various couplings constants and the non-commuting properties of the environment.

The third property is the most relevant for us (and perhaps also less often mentioned). To all orders in $g_{i}$ and for all values of $g_{i}, \Omega_{i}$ (even with arbitrary time dependence), the following identity

$$
\left.i \partial_{t} G_{c}\left(t, t^{\prime} ; p\right)\right|_{t=t^{\prime}}=1,
$$

holds because it corresponds to the ETC of eq. (2.4). The 1 on the $r h s$ is guaranteed by the Hamiltonian character of the evolution of the entire system $\phi+\Psi$. It is therefore this equation which replaces the constancy of the Wronskian that was relevant in the case of free evolution. Eq. (4.4) is crucial for us because the operator $\phi^{d}(t)$ exponentially decays in $t-t_{\text {in }}$ where $t_{\text {in }}$ is the moment when the interactions are turn on, since it is an homogeneous solution of eq. 2.20). Hence the first term in eq. (4.2) decays as $\exp -\gamma\left(t+t^{\prime}-2 t_{i n}\right)$. Therefore at late times with respect to $t_{i n}$ in the units of the inverse decay rate $\gamma^{-1}$, the non-commuting properties of field operator $\hat{\phi}$ are entirely due to those of the environment degrees of freedom, $\hat{\Psi}$.

We now analyze the anti-commutator,

$$
G_{a}\left(t, t^{\prime} ; p\right) \delta^{3}\left(\mathbf{p}-\mathbf{p}^{\prime}\right) \equiv \operatorname{Tr}\left[\rho_{T}\left\{\phi_{\mathbf{p}}(t), \phi_{\mathbf{p}^{\prime}}^{\dagger}\left(t^{\prime}\right)\right\}_{+}\right] .
$$

When the density matrix factorizes as $\rho_{T}=\rho_{\phi} \rho_{\Psi}$ before the interactions are turned on (as it is the case in the "free" vacuum), $G_{a}$ also splits into two terms,

$$
G_{a}\left(t, t^{\prime} ; p\right)=\operatorname{Tr}\left[\rho_{\phi}\left\{\phi^{d}(t), \phi^{d}\left(t^{\prime}\right)\right\}_{+}\right]+\iint d t_{1} d t_{2} G_{r}\left(t, t_{1}\right) G_{r}\left(t^{\prime}, t_{2}\right) N\left(t_{1}, t_{2}\right) .
$$

The first term depends only on the initial state of $\phi$. Similarly, the driven term depends on the state of the environment through the "noise" kernel

$$
N\left(t_{1}, t_{2}\right)=\Sigma_{i} \Sigma_{j} \operatorname{Tr}\left[\rho_{\Psi}\left\{g_{i}\left(t_{1}\right) \Psi_{i}^{o}\left(t_{1}\right), g_{j}\left(t_{2}\right) \Psi_{j}^{o}\left(t_{2}\right)\right\}_{+}\right] .
$$

As for the commutator, in the presence of dissipation, the first term exponentially decays, expressing the progressive erasing on the information contained in the initial state of $\phi$. At late times therefore, as one might have expected, it is the state of $\Psi$ which fixes the anti-commutator of $\phi$. This allows to remove the restriction that initially the density matrices factorizes. If one is interesting by late time behaviour, only $N$ matters.

In brief, two important results are been obtained. First, at late time, the Heisenberg field $\phi$ reduces to its driven term, the second term of eq. (2.19), since both its commutator and anticommutator are determined by those of $\Psi_{i}^{o}$. Second, only two (real) quantities determined by the environment, the set $\Psi_{i}^{o}$ and their couplings, govern the two-point functions of $\phi_{p}$, namely the kernels $D$ and $N$ of eqs. (4.3) and (4.7). Therefore the set of environements (Gaussian or not) possessing the same kernels will give rise to the same 2 pt functions for $\phi$. Hence they should be viewed as forming an equivalent class. The degeneracy can be lifted by considering correlations with observables containing the operators $\Psi_{i}$, or higher order correlations functions of $\phi$ (for non-Gaussian environements), two possibilities we shall not discuss in this paper. 
In order to be able to compute $G_{c}$ and $G_{a}$, two different routes can be adopted. When $g_{i}(p)$ and $\Omega(p)$ are constant, one should work in Fourier transform because the equations can be algebraically solved, in full generality. Instead when $g_{i}(p)$ and/or $\Omega(p)$ are time-dependent, as it will be the case in expanding universes (otherwise the theory would be conformally invariant), it becomes imperative to choose the set of $\Psi_{i}$ 's and their frequency $\Omega_{i}^{2}(p)$ so as to simplify the time dependence of the equations. In the body of the article, we proceed with time dependent approach since it will allow us to combine general covariance and dissipation. We present below the Fourier analysis which is straightforward and well known [20]. We encourage the reader unfamiliar with the treatment of dissipative effects in Quantum Mechanics to read it.

\section{Appendix B :}

\section{Stationary states, and vacuum 2-point functions}

In this Appendix, we provide relationships between $G_{c}, G_{a}$ which always hold in stationary states. In these cases, the Green functions $G_{c}, G_{a}$ and the kernels $D, N$ are functions of $t-t^{\prime}$, and are related to each other in a fundamental way, generally refered as a Fluctuation-Dissipation relation. We briefly explain its origin and its physical implications in the present context. We start the analysis by with the most basic object: the retarded Green function $G_{r}$.

\subsection{The retarded Green function}

The Fourier transform of eq. (2.19) gives

$$
\left(-\omega^{2}+\omega_{p}^{2}\right) \phi_{\mathbf{p}}(\omega)=\Sigma_{i} g_{i}(p) \Psi_{i}^{o}(\mathbf{p}, \omega)+\Sigma_{i} g_{i}^{2}(p) R_{i}^{o}(\omega ; p) \phi_{\mathbf{p}}(\omega),
$$

where

$$
\left.R_{i}^{o}(\omega ; p)=\left(-(\omega+i \varepsilon)^{2}+\Omega_{i}^{2}(p)\right)\right)^{-1},
$$

is the Fourier transform (defined as in eq. (2.10)) of the retarded Green function of $\Psi_{i}$. As usual, its retarded character is enforced by the imaginary prescription of the two poles to lay in the lower half plane $(\varepsilon>0)$. The solution of the above equation is

$$
\phi_{\mathbf{p}}(\omega)=\phi_{\mathbf{p}}^{d}(\omega)+G_{r}(\omega, p) \Sigma_{i} g_{i}(p) \Psi_{i}^{o}(\mathbf{p}, \omega),
$$

where the Fourier transform of the retarded Green function of $\phi$, the solution of eq. (2.20), always takes the form

$$
G_{r}(\omega, p)=\left(-(\omega+i \varepsilon)^{2}+\omega_{p}^{2}+\Sigma_{r}(\omega, p)\right)^{-1} .
$$

All effects of the coupling to the $\Psi_{i}$ 's are thus encoded in the (retarded) self-energy $\Sigma_{r}(\omega, p)$. For Gaussian theories, it is algebraically given by

$$
\Sigma_{r}(\omega, p)=-\Sigma_{i} g_{i}^{2}(p) R_{i}^{o}(\omega, p) .
$$

The dissipative effects are governed by the imaginary part of $\Sigma_{r}(\omega, p)$. In the present case, one has

$$
2 \operatorname{Im} \Sigma_{r}(\omega, p)=-\Sigma_{i} g_{i}^{2}(p) G_{c, i}^{o}(\omega, p)=-D(\omega, p) .
$$


To get the first equality we have used the fact that in stationary states the retarded Green function and the commutator are related by $2 \operatorname{Im} G_{r}(\omega)=G_{c}(\omega)$ for all degrees of freedom, free or interacting. In the second equality, we have introduced $D(\omega)$, the Fourier transform of the kernel of eq. (4.3).

Several observations should be made here. First, from eq. (5.2), we obtain that $D(\omega)$ is proportional to $\Sigma_{i} g_{i}^{2} \delta\left(\omega-\Omega_{i}\right)$. Therefore there is no dissipation for lower frequencies than the lowest value of $\Omega_{i}$. This simply follows from energy conservation. Second, to obtain "true" dissipation, $D(\omega, p)$ should be a continuous function and not a sum of delta. This can only happen when the $\Psi_{i}$ form a dense ensemble. In next Section, we shall thus replace the discrete sum on $i$ by an integral on a continuous variable, $k$. We shall not consider the discrete cases even though these could display interesting properties. Third, from a phenomenological point of view, only $D(\omega, p)$ matters. Hence we cannot separately know what is the spectrum of the environment, which is given by $R_{i}^{o}(\omega, p)$, and what is the coupling strenght $g_{i}^{2}(p)$. This is a good thing, because when working in time-dependent settings, we shall exploit this equivalence to chose the simplest model of $\Psi_{i}$ 's which gives the kernel $D(\omega, p)$ we want.

It is also worth noticing that the dispersive (real) effects are not directly related to $D$ (or $N$ ). These are governed by the even part of $\Sigma_{r}(\omega, p)$ which is given by

$$
\operatorname{Re} \Sigma_{r}(\omega, p)=\int \frac{d \omega^{\prime}}{2 \pi} \frac{D\left(\omega^{\prime}\right)}{\omega-\omega^{\prime}},
$$

where the integral should be understood as a principal value. This integral relationship explains why one often founds that dispersive effects appear before dissipative effects (for increasing $\omega$ ). We also learn that the dispersive models studied in the litterature are incompatible with the above relations since they assume both $\operatorname{Re} \Sigma_{r} \neq 0$ and $\operatorname{Im} \Sigma_{r} \equiv 0$. Therefore these models cannot be viewed as resulting from dynamical processes.

\subsection{Fluctuation-Dissipation relations and vacuum self-energy}

In this subsection, we derive the relationships between $G_{c}, G_{a}$ and $\Sigma_{F}$ which exist in the true (interacting) ground state.

In interacting theories, the only stationary states are thermal states, see e.g. [29]. In these states, the Fourier transform of $D$ and $N$ are related by

$$
\begin{aligned}
N(\omega) & =D(\omega) \operatorname{coth}(\beta \omega / 2), \\
& =D(\omega) \operatorname{sign}(\omega)[2 n(|\omega|)+1] .
\end{aligned}
$$

In the second line, $n(\omega)$ is the Planck distribution. It gives the mean occupation number of $\Psi_{i}^{o}$ quanta as a function of the frequency (measured in the rest frame of the bath). The above relation directly follows from the fact that the individual commutators and anti-commutators of the free fields $\Psi_{i}^{o}$ obey this relation, as any free oscillator would do. It implies that the Fourier transform of $G_{c}$ and $G_{a}$ are also related by

$$
G_{a}(\omega)=G_{c}(\omega) \operatorname{sign}(\omega)[2 n(\omega)+1] .
$$

It should be stressed that this equation is exact, i.e. non-perturbative, and valid for all theories, Gaussian or not. (It indeed directly follows from the cyclic properties of the trace defining $\left.G_{\beta}\left(t, t^{\prime}\right)=\operatorname{Tr}\left[e^{-\beta H_{T}} \phi(t) \phi\left(t^{\prime}\right)\right]\right)$. 
For Gaussian models, there exists an alternative direct verification of eq. (5.9). In fact, it suffices to note that in steady states the decaying terms of eqs. (4.2) and (4.6) play no role, and that the Fourier transform of the driven terms are respectively given by

$$
\begin{aligned}
& G_{c}(\omega)=\left|G_{r}(\omega)\right|^{2} D(\omega), \\
& G_{a}(\omega)=\left|G_{r}(\omega)\right|^{2} N(\omega),
\end{aligned}
$$

since the Fourier transform of the retarded Green function obeys $G_{r}(\omega)=G_{r}^{*}(-\omega)$, see eq. (5.3). Irrespectively of the complexity of $G_{r}$, i.e. irrespectively of the functions $g_{i}(p), \Omega_{i}(p)$ and the set of the $\Psi_{i}$ fields, $G_{c}$ and $G_{a}$ are thus related to each other by the FD relation (5.9).

These universal relations will be relevant for to inflationary models wherein only the ground state contributes. In particular, they imply that in the true vacuum, i.e. when $n(\omega)=0, G_{c}$ and $G_{a}$ are exactly related by $G_{a}(\omega)=G_{c}(\omega) \operatorname{sign}(\omega)$. Hence the Wightman function

$$
G_{W}=\frac{1}{2}\left(G_{c}+G_{a}\right)=G_{c} \theta(\omega)
$$

is determined by the commutator and contains only positive frequency, as in the free vacuum. Equations (5.10, 5.11) also allow to compute the vacuum self-energy of the Feynman Green function in eq. (1.2). For Gaussian models it is given by

$$
2 \operatorname{Im} \Sigma_{F}(\omega)=-D(\omega) \operatorname{sign}(\omega)=2 \operatorname{Re} \Sigma_{i} g_{i}^{2} G_{F, i}^{o}(\omega),
$$

where the $p$ dependence has not been not explicitized, and where we have taken the real part because of the $i$ in the numerator of eq. (1.2). With the last equality we recover the fact that in the vacuum, it is sufficient to consider Feynman Green functions. In non-vacuum states, and in non-stationary situations, this is no longer true, thereby justifying the use of the in - in machinery (also called Schwinger-Keldish formalism).

Before specializing to a specific class of models giving rise to dissipation at high frequency, we make a pause by asking the following important question: What should be known about the $\Psi_{i}$ fields to get eqs. 5.10, 5.11, 5.12) ? We have proven that it is sufficient for the $\Psi_{i}$ 's to be canonical fields, but is it necessary?

The answer is two fold. On one hand, the $\Psi_{i}$ cannot be stochastically fluctuating (i.e. commuting) quantities because this would lead to a violation of eq. (5.8) that would imply the violation of eq. (5.9) and the ETC eq. (4.4). ${ }^{7}$ They cannot be either a combination of quantum and stochastic quantities because this would still lead to a violation of the ETC. Hence they must be built only from quantum (canonical) degrees of freedom.

On the other hand, the $\Psi_{i}$ 's can be composite operators, ${ }^{8}$ i.e. polynomials of some (unknown) canonical fields. Indeed, their commutators would still be all related to their anti-commutators

\footnotetext{
${ }^{7}$ This constitutes the simplest proof that it is inconsistent to couple quantum variables to stochastic (or classical) ones. If one does so, the ETC of the dressed quantum variables will always be dissipated after a time of the order of $\gamma^{-1}$. One can therefore view the experimental evidences for the ETC of some degrees of freedom as a very strong indication that all dynamical variables in our world are quantum mechanical in nature. This line of thought has been used by W. Unruh to prove that gravitational waves must be quantized.

${ }^{8}$ I am grateful to Albert Roura for bringing my attention to this question.
} 
by the FD relation eq. (5.8), and this even though both depend non-linearly on $n(\omega)$ in nonvacuum states. The difference with Gaussian models is that these non-linear operators possess non-vanishing connected higher order correlation functions. Hence, the self-energies $\Sigma_{r}, \Sigma_{F}$ will contain higher a series in powers of $g_{i}^{2}$, and not just a single quadratic term as in eq. (5.5, 5.13). Nevertheless these higher loops corrections preserve the validity of eq. (5.12) in the ground state, as well as that of eqs. (5.10, 5.11) in any thermal state, when properly undertood, i.e. with $D$ now defined by $-2 \operatorname{Im} \Sigma_{r}$ (as the effective dissipation kernel), and $N$ related to it by the FD relation.

In brief, we have reached/recalled the following results. Firstly, the q-number combination $\Xi=\Sigma_{i} g_{i} \Psi_{i}$, the fluctuating source term of $\phi$, must obey the FD relation (5.8). This can either be postulated, or better, be viewed as resulting from the fact that $\Xi$ is entirely made out of quantum degrees of freedom. Secondly, to lowest order term in $g$, the self-energy can be obtained by treating $\Xi$ as a quantum Gaussian variable, whatever its composition may be. Thirdly, when dealing with non-Gaussian theories, once having computed $\Sigma_{r}(\omega)$, the resulting equations for the 2-point functions have the same structure and the same meaning as in Gaussian theories, with $D$ replaced by $-2 \operatorname{Im} \Sigma_{r}$. Therefore, the entire phenomenology of two-point functions can be described with Gaussian settings.

\subsection{The double limit: $g^{2} T \rightarrow \infty$ followed by $g^{2} \rightarrow 0$.}

To perform a phenomenological analysis, we need to understand how the theory behaves in transitory regime from dissipative to free propagation. Similarly, to study primordial spectra in inflation or Hawking radiation, we also need to understand how free motion emerges as the proper frequency get red-shifted. It is therefore relevant to study the behavior of the two-point function in the following double limit.

One first takes $g^{2} T \rightarrow \infty$, where $T=t-t_{i n}, t_{\text {in }}$ being the moment when the interactions are turn on, and $t$ the moment when one studies the field properties. This limit implies that the decaying term in eq. (2.19) plays no role. Therefore, near time $t$, the Heisenberg field $\phi(t)$ is a composite operator which only acts in the Hilbert space of $\Xi$.

Secondly, one considers the "free" limit $g^{2} \rightarrow 0$ of that composite operator. One could naively conclude that $G_{c}$ and $G_{a}$ of eqs. (5.10, 5.11) would vanish since both $D$ and $N$ are proportional to $g^{2}$. However, this is not the case, because the prefactor in these equations, $\left|G_{r}\right|^{2}$, is singular in this second limit. In fact, one verifies that it scales in $1 / g^{2}$ in such a way that, in the (interacting) vacuum, one always recovers

$$
G_{W}(\omega)_{g^{2} \rightarrow 0}=\frac{1}{2 \omega_{p}} 2 \pi \delta\left(\omega-\omega_{p}\right) .
$$

This is the standard vacuum fluctuations of a free oscillator of frequency $\omega_{p}$.

Two important lessons have been reached. First we learned is that even though $\phi$ acts only on the $\Xi$-Hilbert space, when $g^{2} \rightarrow 0$, it behaves as if it were a free mode possessing its own Hilbert space, with no reference to $\Xi$-dynamics. Secondly, in spite of this, the quantum state in the would be Hilbert space is still exactly that of $\Xi$. Therefore, in stationary situations, the only "souvenir" kept by the composite is the equilibrium distribution $n(\omega)$ inherited from its parents.

Let us now emphasize that the above limit is relevant for non-Gaussian models as well. Indeed, in the limit $g^{2} \rightarrow 0$, there will always be a value of $g^{2}$ sufficiently small that the model can 
be well approximated by a Gaussian model. Therefore the behavior of the 2-point functions in the transitory regime from dissipation to free propagation can be analyzed without restriction by studying Gaussian models (at least in the quasi-static limit).

\section{Appendix C:}

\section{Dissipative effects above $\Lambda_{L V}$. The Phenomenology}

We now have all the tools to understand models giving rise to dissipation in the vacuum above a critical energy scale $\Lambda_{L V}$. We proceed in two steps. First, from a purely phenomenological approach, we provide the class of dissipative models wherein the imaginary part of the self-energy is governed by a single term, in analogy with the dispersion relations of eq. (1.5). We then show how to obtain this class of models starting from an action in order to prepare application to inflation and other curved geometries.

If one considers only stationary situations (i.e. static metrics and stationary states), and if one adopts a phenomenological point of view, one can simply choose the function $D(\omega, p)$ entering eq. (5.6) and eq. (5.10) as one wishes. There is indeed no restriction on $D(\omega, p)$ besides its constitutive properties, namely being odd in $\omega$ and giving rise to poles in $G_{r}$ all localized in the lower half $\omega$ plane. In this we have reached our first aim, namely identify how to generalyze the free settings so as to incorporate some arbitrary dissipative effects.

We can thus consider the dispersive models which correspond to those defined by eq. (1.5). They are charaterized by a single term giving rise to dissipation above $\Lambda_{L V}$. In the vacuum, they are fully specified by the imaginary part of the (retarded) self-energy

$$
-\operatorname{Im} \Sigma_{r}^{(n)}(\omega, p)=\frac{\omega}{\Lambda_{L V}} p^{2}\left(\frac{p}{\Lambda_{L V}}\right)^{2 n}=2 \omega \gamma_{n} .
$$

In these models, the decay rate (inverse life time) on the mass shell is

$$
\gamma_{n}=\frac{p}{2}\left(\frac{p}{\Lambda_{L V}}\right)^{2 n+1} .
$$

To verify it, assuming that $\operatorname{Re} \Sigma_{r}=0$, the two poles of $G_{r}(\omega)$ in eq. (5.4) are located in

$$
\omega_{ \pm}(p)= \pm \sqrt{\omega_{p}^{2}-\gamma^{2}}-i \gamma
$$

From this, by inverse Fourier transform $G_{r}(\omega)$, one obtains that the decay rate is indeed $\gamma$ in the underdamped regime, for $\omega_{p}^{2}>\gamma^{2}$. In the overdamped regime, for $\gamma^{2}>\omega_{p}^{2}$, the decay rates of the two independent solutions of $G_{r}^{-1} \phi_{d}=0$ are $\Gamma_{ \pm}=\gamma \pm \sqrt{\gamma^{2}-\omega_{p}^{2}}$.

One thus have the following behavior as $p$ grows. For $p \ll \Lambda_{L V}, \omega_{ \pm} \simeq p$, and one has a free propagation which is slightly damped with a life time in the units of the frequency given by $\left(\Lambda_{L V} / \omega\right)^{n+1} \gg 1$. Instead the opposite regime of high momenta $p \gg \Lambda_{L V}$, deep in the overdamped regime, the two roots $\omega_{ \pm}$are real and the notion of propagation (in space-time) is completely absent. In anticipation to what will occur in inflation or in black hole physics, we invite the reader to study the migration of the poles of $G_{r}$ when extrapoling backwards in time a mode, i.e. as $p$ increases. (Remember that the physical momentum of a mode in cosmology is $p(t)=p_{o} / a(t)$ 
where $p_{o}$ is the norm of the conserved comoving wave vector, whereas near a black hole horizon one finds $p(r)=\omega / x$ where $x=r-r_{S}$ is the proper distance from the horizon measured in a freely falling frame, and $\omega$ the conserved Killing frequency measured asymptotically.)

One could of course generalize the above class by considering in eq. (6.1) polynomials in $p$ dimensionalized by different UV scales. However, unless fine tuning, the phenomenology of the transition from the IR dissipation-free sector to the dissipative sector will be dominated a single term. One should also consider the possibility that $\operatorname{Im} \Sigma$ strictly vanishes below a certain frequency $\Omega_{1}$. This would be the case when the spectrum of the $\Psi$ fields possesses such a gap, see the remarks after eq. (5.6).

Having the phenomenology of dissipative unitary models under control (with dispersive and dissipative related by Kramers relations, see eq. (5.7p) one could confront particle and astro-particle physics data and put lower bounds on $\Lambda_{L V}$ for each $n$, in analogy with what was done for (pure) dispersion in [2].

\section{References}

[1] T. Jacobson, Phys. Rev. D 44 (1991) 1731.

[2] T. Jacobson, S. Liberati and D. Mattingly, Annals Phys. 321 (2006) 150.

[3] R. Parentani, Nucl. Phys. B 492 (1997) 475.

[4] E. Witten, Nucl. Phys. B 311 (1988) 46.

[5] R. Parentani, Nucl. Phys. B 492 (1997) 501; S. Massar and R. Parentani, Nucl. Phys. B 513 (1998) 375, and Phys. Rev. D 59 (1999) 123519.

[6] N. D. Birrell and P. C. W. Davies, "Quantum fields in curved space," Cambridge University Press (1982).

[7] R. M. Wald, "Quantum field theory in curved space-time and black hole thermodynamics," Chicago, USA: Univ. Pr. (1994).

[8] B. Z. Foster and T. Jacobson, JHEP 0408 (2004) 024.

[9] A. Kempf, Phys. Rev. D 63 (2001) 083514.

[10] V. F. Mukhanov, H. A. Feldman and R. H. Brandenberger, Phys. Rept. 215 (1992) 203.

[11] R. Brout, S. Massar, R. Parentani, and Ph. Spindel, Phys. Rep. 260 (1995) 329.

[12] W. G. Unruh, Phys. Rev. D 51 (1995) 2827.

[13] T. Jacobson, Phys. Rev. D 48 (1993) 728.

[14] R. Brout, S. Massar, R. Parentani and P. Spindel, Phys. Rev. D 52 (1995) 4559.

[15] J. Martin and R. H. Brandenberger, Phys. Rev. D 63 (2001) 123501.

[16] J. C. Niemeyer, Phys. Rev. D 63 (2001) 123502.

[17] M. V. Libanov and V. A. Rubakov, JCAP 0509 (2005) 005.

[18] M. V. Libanov and V. A. Rubakov, Phys. Rev. D 72 (2005) 123503.

[19] T. Jacobson, Phys. Rev. D 53 (1996) 7082. 
[20] P. C. Aichelburg and R. Beig, Annals of Physics, 98 (1976) 264.

[21] D. J. Raine, D. W. Sciama and P. G. Grove, Proc. R. Soc. A 435 (1991) 205.

[22] S. Massar, R. Parentani and R. Brout, Class. Quant. Grav. 10 (1993) 385.

[23] C. Gardiner and P. Zoller, "Quantum Noise," Springer (2000).

[24] W. G. Unruh and W. H. Zurek, Phys. Rev. D 40 (1989) 1071.

[25] D. Arteaga, arXiv:0711.0293 [quant-ph].

[26] B. S. DeWitt, Phys. Rept. 19 (1975) 295.

[27] W. G. Unruh, Phys. Rev. Lett. 46 (1981) 1351.

[28] J. C. Niemeyer and R. Parentani, Phys. Rev. D 64 (2001) 101301.

[29] J. R. Anglin, arXiv:hep-th/9210034.

[30] T. Jacobson and D. Mattingly, Phys. Rev. D 61 (2000) 024017.

[31] R. Balbinot, A. Fabbri, S. Fagnocchi and R. Parentani, Riv. Nuovo Cim. 28 (2005) 1.

[32] J. C. Niemeyer, R. Parentani and D. Campo, Phys. Rev. D 66 (2002) 083510.

[33] D. S. Salopek and J. M. Stewart, Phys. Rev. D 47 (1993) 3235.

[34] T. Jacobson and R. Parentani, Phys. Rev. D 76, 024006 (2007). 